\title{
A high-order staggered finite-element vertical discretization for non-hydrostatic atmospheric models
}

\author{
Jorge E. Guerra and Paul A. Ullrich \\ Department of Land, Air and Water Resources, University of California, Davis, One Shields Ave., Davis, CA 95616, USA \\ Correspondence to: Jorge E. Guerra (jeguerra@ucdavis.edu)
}

Received: 18 December 2015 - Published in Geosci. Model Dev. Discuss.: 19 January 2016

Revised: 10 May 2016 - Accepted: 11 May 2016 - Published: 1 June 2016

\begin{abstract}
Atmospheric modeling systems require economical methods to solve the non-hydrostatic Euler equations. Two major differences between hydrostatic models and a full non-hydrostatic description lies in the vertical velocity tendency and numerical stiffness associated with sound waves. In this work we introduce a new arbitrary-order vertical discretization entitled the staggered nodal finiteelement method (SNFEM). Our method uses a generalized discrete derivative that consistently combines the discontinuous Galerkin and spectral element methods on a staggered grid. Our combined method leverages the accurate wave propagation and conservation properties of spectral elements with staggered methods that eliminate stationary $(2 \Delta x)$ modes. Furthermore, high-order accuracy also eliminates the need for a reference state to maintain hydrostatic balance. In this work we demonstrate the use of high vertical order as a means of improving simulation quality at relatively coarse resolution. We choose a test case suite that spans the range of atmospheric flows from predominantly hydrostatic to nonlinear in the large-eddy regime. Our results show that there is a distinct benefit in using the high-order vertical coordinate at low resolutions with the same robust properties as the low-order alternative.
\end{abstract}

\section{Introduction}

The accurate representation of vertical wave motion is essential for models of the atmosphere. The vertical coordinate for the non-hydrostatic fluid equations has traditionally been discretized in the Eulerian frame via a second-order Charney-Phillips (Charney and Phillips, 1953) or Lorenz grid (Arakawa and Moorthi, 1988), or via Lagrangian lay- ers such as in Lin (2004). However, little work has been undertaken to develop high-order vertical discretizations due to a number of outstanding issues. First, higher-order generalizations must somehow incorporate the no-flux boundary conditions at the model bottom and top without loss of accuracy, especially near the surface where accurate treatment of dynamics is paramount. Second, as observed by Thuburn and Woollings (2005), Thuburn (2006), and Toy and Randall (2007), the choice of vertical coordinate (whether height based, mass based or entropy based) implies an optimal vertical staggering of prognostic variables for maintaining correct behavior for wave motions relevant to the atmosphere. Third, unstaggered discretizations (that is, discretizations where all prognostic variables are stored on model levels) possess stationary computational modes, which represent gross errors in the dispersion properties of the solution (Melvin et al., 2012; Ullrich, 2014b). As in the horizontal, unstaggered Finite Element Method (FEM) leads to waves with zero phase speed in the limit as the wavelength tends to $2 \Delta x$, where $\Delta x$ is the average grid spacing between degrees of freedom. However, unlike the horizontal, these wave modes can be dramatically enhanced by an implicit treatment of the vertical at high Courant number.

This paper describes a new discretization for the vertical that combines the accuracy of finite-element methods with the desirable wave propagation properties of staggered methods. This method of vertical discretization was originally described in Ullrich and Guerra (2015), but tested using a modified set of equations and validated with a single test case. Here we extend this approach, referred to as the staggered nodal finite-element method (SNFEM), in a similar framework. Notably, this formulation is sufficiently general to be compatible with essentially any form of the fluid equations. 
The SNFEM discretization can be easily composed in differential form using interpolation and differentiation operators built in accordance with the discontinuous Galerkin and spectral element discretizations that arise from the flux reconstruction method of Huynh (2007) (see Table 1).

Our staggered method is similar to the mixed finiteelement formulations of Cotter and Thuburn $(2012,2014)$ where different functional spaces are used on the prognostic fields in order to achieve desirable wave propagation and conservation properties. The SNFEM utilizes different polynomial spaces based on continuous and discontinuous grids to achieve staggered configurations. The use of SNFEM is natural for vertical discretizations, as no-flux conditions are easily imposed on top and bottom boundaries in the general finite-element framework (Zienkiewicz et al., 2005) without loss of accuracy. Further, SNFEM inherits the mimetic properties of the spectral element method so the vertical operator will automatically conserve both mass and discrete linear energy. The objectives of this paper are as follows:

1. To introduce our approach for the construction of a generalized, staggered, variable order-of-accuracy, finite-element vertical discretization. We emphasize discretization of the non-conservative differential form of the Navier-Stokes equations (in vector invariant or socalled Clark form), which is independent of coordinate system.

2. To validate the implementation of this discretization within the Tempest framework using a selection of test cases in Cartesian geometry through a range of horizontal scales from 1 to $1000 \mathrm{~km}$.

3. To determine the qualitative and quantitative effect of vertical order of accuracy on solutions by conducting validation experiments at coarse resolutions relative to finer reference solutions. We consider the effects of Lorenz (LOR) and Charney-Phillips (CPH) staggering both in the interior flow and at the lower boundary.

4. To determine whether a high-order vertical discretization greatly improves the simulation quality, and consequently to recommend whether there is an optimal order-of-accuracy that provides the best tradeoff between accuracy and computational cost.

To assess the performance of SNFEM, this discretization has been implemented in the spectral element Tempest model (Ullrich, 2014a) and run through a suite of mesoscale test cases. The test cases are as follows: baroclinic instability in a 3-D Cartesian channel of Ullrich et al. (2015), uniform flow over the mountain of Schär et al. (2002), the density current of Straka et al. (1993), and rising thermal convective bubble tests as given in Giraldo and Restelli (2008). While not exhaustive, this validation suite is intended to show the treatment of waves, nonlinear vertical transport, and nearboundary dynamics corresponding to a high-order vertical coordinate with and without the influence of topography. Therefore, the objectives of this paper are as follows:

We will show that a high-order vertical discretization at coarse resolution more accurately approximates the reference solution relative to the low vertical-order alternative when total count of degrees of freedom is kept constant. Since the interpolation and derivative operators in the finiteelement approach are easily expressed as linear matrix operators, there is minimal cost in adjusting the order-of-accuracy. We will present control experiments in Sect. 4 where only the resolution and vertical order-of-accuracy vary. We leave the rigorous analysis of staggered wave modes and discrete energy conservation using the interpolation/differentiation operators for subsequent work.

The remainder of this manuscript is as follows: Sect. 2 describes the non-hydrostatic equations of fluid motion on an arbitrary coordinate frame. Section 3 describes the discrete form of these equations, including the spectral element horizontal discretization, the operators used by the SNFEM vertical discretization and the time-stepping scheme employed. In Sect. 4, we describe the test case suite and discuss the corresponding model results. The summary and conclusions follow in Sect. 5.

\section{The non-hydrostatic equations of fluid motion}

In an arbitrary coordinate frame $(\alpha, \beta, \xi)$, the vector velocity can be written as

$\boldsymbol{u}=u^{\alpha} \boldsymbol{g}_{\alpha}+u^{\beta} \boldsymbol{g}_{\beta}+u^{\xi} \boldsymbol{g}_{\xi}$,

where $\boldsymbol{g}_{i}(i \in\{\alpha, \beta, \xi\})$ are the local coordinate basis vectors and $u^{i}$ are the contravariant velocity components. The associated covariant components are

$u_{\alpha}=\boldsymbol{u} \cdot \boldsymbol{g}_{\alpha}, u_{\beta}=\boldsymbol{u} \cdot \boldsymbol{g}_{\beta}, u_{\xi}=\boldsymbol{u} \cdot \boldsymbol{g}_{\xi}$.

Covariant components can be obtained in terms of contravariant components via contraction with the covariant metric $g_{i j}=\boldsymbol{g}_{i} \cdot \boldsymbol{g}_{j}$,

$u_{i}=g_{i \alpha} u^{\alpha}+g_{i \beta} u^{\beta}+g_{i \xi} u^{\xi}$.

The reverse operation uses the contravariant metric $g^{i j}$, defined as the matrix inverse of the covariant metric. Contraction of the covariant components with the contravariant metric returns the contravariant vector components,

$u^{i}=g^{i \alpha} u_{\alpha}+g^{i \beta} u_{\beta}+g^{i \xi} u_{\xi}$.

The volume element $J$ is computed in terms of the covariant metric as

$J=\sqrt{\operatorname{det} g_{i j}}$.

Using covariant horizontal velocity components, vertical velocity, potential temperature $\theta$, and dry air density $\rho$ 
Table 1. Composition of interpolation $\mathcal{I}$ and differentiation $\mathcal{D}$ operators for several choices of staggering, including collocated spectral elements (SE), SNFEM with Lorenz staggering (SNFEM-LOR), and SNFEM with Charney-Phillips staggering (SNFEM-ChP). Script $i$ denotes variables defined on interfaces (Gauss-Lobatto nodes) and $n$ represents variables defined on model levels (Gauss nodes). For operator $\mathcal{I}$ and $\mathcal{D}$, the subscript denotes the target $(i$ or $n)$ and the superscript denotes the origin.

\begin{tabular}{cc|ccc}
\hline \multirow{2}{*}{ Variable } & Term & SE $\left(\rho_{i}, \theta_{i}, w_{i}\right)$ & SNFEM-LOR $\left(\rho_{n}, \theta_{n}, w_{i}\right)$ & SNFEM-ChP $\left(\rho_{n}, \theta_{i}, w_{i}\right)$ \\
\hline$u, v$ & $\Pi$ & $\Pi_{i}\left(\rho_{i}, \theta_{i}\right)$ & $\Pi_{n}\left(\rho_{n}, \theta_{n}\right)$ & $\Pi_{n}\left(\rho_{n}, \mathcal{I}_{n}^{i} \theta_{i}\right)$ \\
$\theta$ & $u^{\xi} \frac{\partial \theta}{\partial \xi}$ & $\left(u_{i}^{\xi}\right) \mathcal{D}_{i}^{i} \theta_{i}$ & $\left(\mathcal{I}_{n}^{i} u_{i}^{\xi}\right)\left(\mathcal{D}_{n}^{n} \theta\right)$ & $\left(u_{i}^{\xi}\right)\left(\mathcal{D}_{i}^{i} \theta_{i}\right)$ \\
$w$ & $\theta \frac{\partial \Pi}{\partial \xi}$ & $\theta_{i} \mathcal{D}_{i}^{i} \Pi_{i}$ & $\left(\mathcal{I}_{n}^{i} \theta_{n}\right)\left(\mathcal{D}_{i}^{n} \Pi_{n}\right)$ & $\theta_{i}\left(\mathcal{D}_{i}^{n} \Pi_{n}\right)$ \\
$\rho$ & $\frac{1}{J} \frac{\partial}{\partial \xi}\left(J \rho u^{\xi}\right)$ & $\frac{1}{J_{i}} \mathcal{D}_{i}^{i}\left(J_{i} \rho_{i} u_{i}^{\xi}\right)$ & $\frac{1}{J_{n}} \mathcal{D}_{n}^{i}\left[J_{i}\left(\mathcal{I}_{i}^{n} \rho_{n}\right) u_{i}^{\xi}\right]$ & $\frac{1}{J_{n}} \mathcal{D}_{n}^{i}\left[J_{i}\left(\mathcal{I}_{i}^{n} \rho_{n}\right) u_{i}^{\xi}\right]$ \\
\hline
\end{tabular}

as prognostic variables, the Euler equations with shallowatmosphere approximation can be written with an arbitrary coordinate frame as

$$
\begin{aligned}
\frac{\partial u_{\alpha}}{\partial t} & =-\frac{\partial}{\partial \alpha}(K+\Phi)-\theta \frac{\partial \Pi}{\partial \alpha}+(\boldsymbol{\eta} \times \boldsymbol{u})_{\alpha}, \\
\frac{\partial u_{\beta}}{\partial t} & =-\frac{\partial}{\partial \beta}(K+\Phi)-\theta \frac{\partial \Pi}{\partial \beta}+(\boldsymbol{\eta} \times \boldsymbol{u})_{\beta}, \\
\left(\frac{\partial r}{\partial \xi}\right) \frac{\partial w}{\partial t} & =-\frac{\partial}{\partial \xi}(K+\Phi)-\theta \frac{\partial \Pi}{\partial \xi}+(\boldsymbol{\eta} \times \boldsymbol{u})_{\xi}, \\
\frac{\partial \theta}{\partial t} & =-u^{\alpha} \frac{\partial \theta}{\partial \alpha}-u^{\beta} \frac{\partial \theta}{\partial \beta}-u^{\xi} \frac{\partial \theta}{\partial \xi}, \\
\frac{\partial \rho}{\partial t} & =-\frac{1}{J} \frac{\partial}{\partial \alpha}\left(J \rho u^{\alpha}\right)-\frac{1}{J} \frac{\partial}{\partial \beta}\left(J \rho u^{\beta}\right) \\
& -\frac{1}{J} \frac{\partial}{\partial \xi}\left(J \rho u^{\xi}\right) .
\end{aligned}
$$

The vertical velocity $w$ is closely related to $u_{\xi}$ via

$w=\left|g_{\xi}\right|^{-1} u \xi$

The specific Kinetic energy is

$K=\frac{1}{2}\left(u_{\alpha} u^{\alpha}+u_{\beta} u^{\beta}+u_{\xi} u^{\xi}\right)$,

while the geopotential function $\Phi$ is given by the product of gravitational acceleration (constant) with the elevation coordinate $r(\xi)$.

$\Pi=c_{p}\left(\frac{p_{0}}{p}\right)^{R_{d} / c_{p}}=c_{p}\left(\frac{R_{d} \rho \theta}{p_{0}}\right)^{R_{d} / c_{v}}$

Here $p_{0}$ denotes the constant reference pressure, $R_{d}$ is the ideal gas constant, and $c_{v}$ and $c_{p}$ refer to the specific heat capacity at constant volume and pressure, respectively. The absolute vorticity vector is given by

$\eta=\zeta+\omega$, where the relative vorticity vector is

$$
\begin{aligned}
\boldsymbol{\zeta} & =\frac{1}{J}\left[\left(\frac{\partial u_{\xi}}{\partial \beta}-\frac{\partial u_{\beta}}{\partial \xi}\right) \boldsymbol{g}_{\alpha}+\left(\frac{\partial u_{\alpha}}{\partial \xi}-\frac{\partial u_{\xi}}{\partial \alpha}\right) \boldsymbol{g}_{\beta}\right. \\
& \left.+\left(\frac{\partial u_{\beta}}{\partial \alpha}-\frac{\partial u_{\alpha}}{\partial \beta}\right) \boldsymbol{g}_{\xi}\right],
\end{aligned}
$$

and, under the shallow-atmosphere approximation, the planetary vorticity vector is

$\omega=f(\partial r / \partial \xi)^{-1} \boldsymbol{g}_{\xi}$.

Consequently, the rotational terms in the equation of motion take the form

$$
\begin{aligned}
& (\boldsymbol{\eta} \times \boldsymbol{u})_{\alpha}=J\left[u^{\beta}\left(\boldsymbol{\omega}^{\xi}+\zeta^{\xi}\right)-u^{\xi} \zeta^{\beta}\right], \\
& (\boldsymbol{\eta} \times \boldsymbol{u})_{\beta}=J\left[u^{\xi} \zeta^{\alpha}-u^{\alpha}\left(\boldsymbol{\omega}^{\xi}+\zeta^{\xi}\right)\right], \\
& (\boldsymbol{\eta} \times \boldsymbol{u})_{\xi}=J\left[u^{\alpha} \zeta^{\beta}-u^{\beta} \boldsymbol{\zeta}^{\alpha}\right] .
\end{aligned}
$$

Note that this formulation does not specify a coordinate system. Consequently, these equations can be used for either Cartesian or spherical geometry. To account for topography, terrain-following $\sigma$-coordinates are imposed by defining the radius $r=r(\alpha, \beta, \xi)$ so that $r(\alpha, \beta, 0)$ is coincident with the surface. For example, Gal-Chen and Somerville (1975) coordinates arise from the choice

$r(\alpha, \beta, \xi)=\xi\left[r_{\text {top }}-r_{\mathrm{s}}(\alpha, \beta)\right]+r_{\mathrm{e}}+r_{\mathrm{s}}(\alpha, \beta)$,

where $r_{\text {top }}$ denotes the model height and $r_{\mathrm{s}}(\alpha, \beta)$ denotes the surface elevation from the mean Earth radius $r_{\mathrm{e}}$. In Cartesian coordinates $r$ simply maps to the elevation $z$ while neglecting the mean radius $r_{\mathrm{e}}$ in Eq. (20). The symmetric covariant and contravariant metric tensors in the $(\alpha, \beta, r)$ Cartesian system 
are written as

$$
\begin{aligned}
& g^{i j}= \\
& \left(\begin{array}{rrrr}
1 & 0 & & -\left(\frac{\partial r}{\partial \xi}\right)^{-1}\left(\frac{\partial r}{\partial \alpha}\right) \\
& 1 & -\left(\frac{\partial r}{\partial \xi}\right)^{-1}\left(\frac{\partial r}{\partial \beta}\right) \\
& & \left(\frac{\partial r}{\partial \xi}\right)^{-2}\left[1+\left(\frac{\partial r}{\partial \alpha}\right)^{2}+\left(\frac{\partial r}{\partial \beta}\right)^{2}\right]
\end{array}\right) \\
& : i, j=(\alpha, \beta, \xi)
\end{aligned}
$$

and

$$
\begin{aligned}
& g_{i j}=\left(\begin{array}{cc}
{\left[1+\left(\frac{\partial r}{\partial \alpha}\right)^{2}\right]} & \frac{\partial r}{\partial \alpha} \frac{\partial r}{\partial \beta} \frac{\partial r}{\partial \xi} \\
{\left[1+\left(\frac{\partial r}{\partial \beta}\right)^{2}\right]} & \frac{\partial r}{\partial \beta} \frac{\partial r}{\partial \xi} \\
& \left(\frac{\partial r}{\partial \xi}\right)^{2}
\end{array}\right) \\
& : i, j=(\alpha, \beta, \xi) \text {. }
\end{aligned}
$$

We note that in this framework, the discretization is decoupled from the grid definition. As such, Tempest is designed to target flows on the sphere and in Cartesian domains simultaneously with or without terrain. This is convenient in the analysis, implementation, and validation of the numerical techniques that follow. We focus our validation on Cartesian cases and will address test cases on the sphere in a subsequent publication based on the same discretization framework. Lastly, derivatives of the vertical coordinate in $\alpha$ and $\beta$ are evaluated using the discrete derivative operators developed in the next section while the vertical gradient of coordinate surfaces can easily be obtained analytically from Eq. (20).

\section{Discretization}

\subsection{Horizontal discretization}

The horizontal discretization of Eqs. (6)-(10) follows the continuous element formulation of Ullrich (2014a), which is analogous to earlier efforts with spectral elements (Giraldo and Rosmond, 2004; Taylor and Fournier, 2010; Dennis et al., 2011; Giraldo et al., 2013) with coordinate information completely contained in the definition of the metric tensors Eqs. (21) and (22).

\subsection{Vertical discretization}

Each vertical column consists of $n_{\mathrm{ve}}$ nodal finite elements, indexed $a \in\left\{0, \ldots, n_{\mathrm{ve}}-1\right\}$. Throughout this manuscript, all vertical indices are assumed to increase with altitude. Within (a)

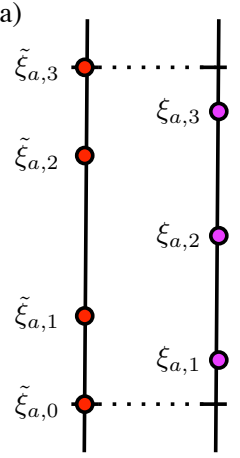

(b)

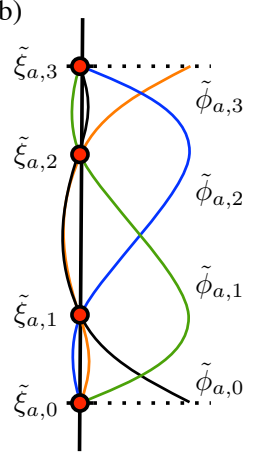

(c)

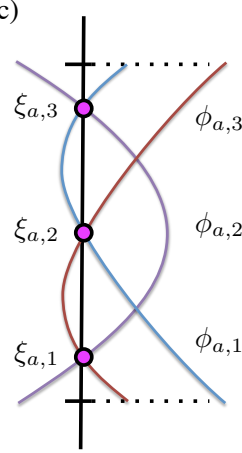

Figure 1. (a) Vertical placement of (left) Gauss-Lobatto nodes and (right) Gauss nodes within a vertical element with $n_{\mathrm{vp}}=3$. (b) Basis functions $\widetilde{\phi}_{a, k}$ for Gauss-Lobatto nodes within element $a$. (c) Basis functions $\phi_{a, k}$ for Gauss nodes within element $a$.

each element, levels are placed at the $n_{\mathrm{vp}}$ Gaussian quadrature nodes and interfaces at $n_{\mathrm{vp}}+1$ Gauss-Lobatto quadrature nodes, leading to a staggering of levels and interfaces. With vertical coordinate $\xi$, the location of model levels denoted $\xi_{a, k}$ with $k \in\left\{0, \ldots, n_{\mathrm{vp}}-1\right\}$ and model interfaces denoted $\widetilde{\xi}_{a, k}$ with $k \in\left\{0, \ldots, n_{\mathrm{vp}}\right\}$. Each finite element is then bounded within the interval $\left[\xi_{a, 0}, \widetilde{\xi}_{a, n_{\mathrm{vp}}}\right]$ with two associated sets of basis functions - one over model levels, denoted by the set $\phi_{a}=\left\{\phi_{a, j} \mid j=0, \ldots, n_{\mathrm{vp}}-1\right\}$ that includes characteristic polynomials of degree $n_{\mathrm{vp}}-1$, and one over model interfaces, denoted by the set $\widetilde{\boldsymbol{\phi}}_{a}=\left\{\widetilde{\phi}_{a}, j \mid j=0, \ldots, n_{\mathrm{vp}}-1\right\}$ that includes characteristic polynomials of degree $n_{\mathrm{vp}}$. A depiction of the vertical staggering associated with levels and interfaces is given in Fig. 1, along with basis functions in each case. A scalar field $q(\xi, t)$ can then be written approximately, either as a linear combination of basis functions on levels,

$q(\xi, t) \approx \sum_{a=0}^{n_{\mathrm{ve}}-1} \sum_{j=0}^{n_{\mathrm{vp}}-1} q_{a, j}(t) \phi_{a, j}(\xi)$

or on interfaces,

$q(\xi, t) \approx \sum_{a=0}^{n_{\mathrm{ve}}-1} \sum_{j=0}^{n_{\mathrm{vp}}} \widetilde{q}_{a, j}(t) \widetilde{\phi}_{a, j}(\xi)$.

For the remainder of this manuscript we will use script $n$ to denote variables stored on model levels and script $i$ to denote variables stored on interfaces.

\subsubsection{Interpolation operators}

Note that Eqs. (23) and (24) are not equivalent discretizations since Eq. (23) cannot represent polynomials of degree $n_{\mathrm{vp}}$ and Eq. (24) cannot represent fields that are discontinuous at element interfaces. Nonetheless, we can define interpolation operators between these fields via $\mathcal{I}_{i}^{n}$, representing interpo- 
lation from levels to interfaces, and $\mathcal{I}_{n}^{i}$, representing interpolation from interfaces to nodes. First, interpolation from interfaces to levels is defined as

$\left(\mathcal{I}_{n}^{i} \widetilde{q}\right)_{a, k}=\sum_{j=0}^{n_{\mathrm{vp}}} \widetilde{q}_{a, j}(t) \widetilde{\phi}_{a, j}\left(\xi_{a, k}\right)$.

To define the interpolant from levels to interfaces, a twostep procedure is employed. Since basis functions on levels are discontinuous, we define the left and right interpolants at element boundaries as

$$
\begin{aligned}
\left(\mathcal{I}_{L}^{n} q\right)_{a, 0} & =\sum_{j=0}^{n_{\mathrm{vp}}-1} q_{a, j} \phi_{a, j}\left(\widetilde{\xi}_{a, 0}\right), \\
\left(\mathcal{I}_{R}^{n} q\right)_{a, n_{\mathrm{vp}}-1} & =\sum_{j=0}^{n_{\mathrm{vp}}-1} q_{a, j} \phi_{a, j}\left(\widetilde{\xi}_{a, n_{\mathrm{vp}}-1}\right)
\end{aligned}
$$

and then define the total interpolant as

$$
\begin{aligned}
& \left(\mathcal{I}_{i}^{n} q\right)_{a, k}= \\
& \begin{cases}\sum_{j=0}^{n_{\mathrm{vp}}-1} q_{a, j} \phi_{a, j}\left(\widetilde{\xi}_{a, k}\right) & \text { if } 0<k<n_{\mathrm{vp}}, \\
\frac{1}{2}\left(\mathcal{I}_{R}^{n} q\right)_{a-1, n_{\mathrm{vp}}-1}+\frac{1}{2}\left(\mathcal{I}_{L}^{n} q\right)_{a, 0} & \text { if } k=0, \\
\frac{1}{2}\left(\mathcal{I}_{R}^{n} q\right)_{a, n_{\mathrm{vp}}-1}+\frac{1}{2}\left(\mathcal{I}_{L}^{n} q\right)_{a+1,0} & \text { if } k=n_{\mathrm{vp} .}\end{cases}
\end{aligned}
$$

These interpolation operators can also be obtained from equivalence via the variational (weak) form. At model interfaces, the accuracy of Eq. (27) degrades for unequally spaced finite elements. For the case of stacked finite elements with unequal thickness $\Delta \xi_{a}=\widetilde{\xi}_{a, n_{\mathrm{vp}}}-\widetilde{\xi}_{a, 0}$, a more accurate formula can be obtained from

$\left(\mathcal{I}_{i}^{n} q\right)_{a, 0}=\frac{\Delta \xi_{a}^{n_{\mathrm{vp}}}\left(\mathcal{I}_{R}^{n} q\right)_{a-1, n_{\mathrm{vp}}-1}+\Delta \xi_{a-1}^{n_{\mathrm{vp}}}\left(\mathcal{I}_{L}^{n} q\right)_{a, 0}}{\Delta \xi_{a}^{n_{\mathrm{vp}}}+\Delta \xi_{a-1}^{n_{\mathrm{vp}}}}$,

which arises on noting that the one-sided interpolant has error $O\left(\Delta \xi_{a}^{n_{\mathrm{vp}}}\right)$.

\subsubsection{Differentiation operators}

Differentiation is required for all combinations of model levels and interfaces: $\mathcal{D}_{i}^{i}$ represents differentiation from interfaces to interfaces, $\mathcal{D}_{n}^{i}$ represents differentiation from interfaces to levels, $\mathcal{D}_{n}^{n}$ denotes differentiation from levels to levels, and $\mathcal{D}_{i}^{n}$ denotes differentiation from levels to interfaces. A depiction of the behavior of these derivative operators is shown in Fig. 2.

Differentiation from interfaces to levels is obtained by simply differentiating Eq. (25),

$\left(\mathcal{D}_{n}^{i} q\right)_{a, k}=\sum_{j=0}^{n_{\mathrm{vp}}} \widetilde{q}_{j} \frac{\partial \widetilde{\phi}_{j}}{\partial \xi}\left(\xi_{a, k}\right)$.

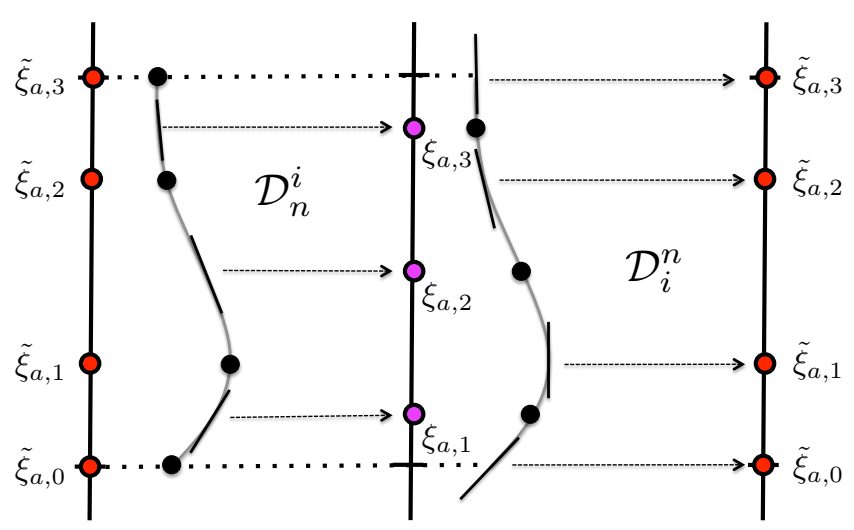

Figure 2. A depiction of the derivative operators $\mathcal{D}_{n}^{i}$ and $\mathcal{D}_{i}^{n}$, which remap from interfaces to levels and levels to interfaces, respectively. The gray line depicts a typical field variable within element $a$ that emerges from the expansion (left) (Eq. 24) or (center) (Eq. 23).

This works in practice as there is an exact mapping from derivatives of the continuous polynomial space (over interfaces) to the discontinuous polynomial space (over levels).

Differentiation from levels to levels is computed via the composed operator

$\mathcal{D}_{n}^{n} q=\mathcal{D}_{n}^{i} \mathcal{I}_{i}^{n} q$,

where boundary conditions, such as the no-flux condition $\left(u^{\xi}=0\right)$ at the top and bottom, are enforced after application of the interpolation operator.

Differentiation from interfaces to interfaces requires averaging the one-sided derivatives at element interfaces, but is otherwise simply the derivative of Eq. (25) on the element interior,

$$
\begin{aligned}
& \left(\mathcal{D}_{i}^{i} q\right)_{a, k}= \\
& \begin{cases}\frac{1}{2}\left(\sum_{j=0}^{n_{\mathrm{vp}}} \widetilde{q}_{a, j} \frac{\partial \widetilde{\phi}_{a, j}}{\partial \xi}\left(\widetilde{\xi}_{a, k}\right)\right. & \\
\left.\quad+\sum_{j=0}^{n_{\mathrm{vp}}} \widetilde{q}_{a-1, j} \frac{\partial \widetilde{\phi}_{a-1, j}}{\partial \xi}\left(\widetilde{\xi}_{a, k}\right)\right) & \text { if } k=0, \\
\sum_{j=0}^{n_{\mathrm{vp}}} \widetilde{q}_{a, j} \frac{\partial \widetilde{\phi}_{a, j}}{\partial \xi}\left(\widetilde{\xi}_{a, k}\right) & \text { if } 0<k<n_{\mathrm{vp}}, \\
\frac{1}{2}\left(\sum_{j=0}^{n_{\mathrm{vp}}} \widetilde{q}_{a, j} \frac{\partial \widetilde{\phi}_{a, j}}{\partial \xi}\left(\widetilde{\xi}_{a, k}\right)\right. & \\
\left.\quad+\sum_{j=0}^{n_{\mathrm{vp}}} \widetilde{q}_{a+1, j} \frac{\partial \widetilde{\phi}_{a+1, j}}{\partial \xi}\left(\widetilde{\xi}_{a, k}\right)\right) & \text { if } k=n_{\mathrm{vp} .} .\end{cases}
\end{aligned}
$$

Differentiation from levels to interfaces $\left(\mathcal{D}_{i}^{n}\right)$ should not be defined via the composition $\mathcal{D}_{i}^{i} \mathcal{I}_{i}^{n}$, since this procedure would introduce a non-zero null space that can trigger an unphysical computational mode in the discrete equations. Instead we define $\mathcal{D}_{i}^{n}$ using the robust differentiation technique discussed in Ullrich (2014a), based on the flux reconstruction 
methods of Huynh (2007). This strategy leads to the discrete operator

$$
\begin{aligned}
\left(\mathcal{D}_{i}^{n} q\right)_{a, k}= & \left.\left(\hat{\mathcal{D}}_{i}^{n} q\right)_{a, k}+\frac{1}{2} \frac{\mathrm{d} G_{R}}{\mathrm{~d} \xi} \widetilde{\xi}_{a, k}\right)\left[\left(\mathcal{I}_{L}^{n} q\right)_{a+1, k}-\left(\mathcal{I}_{R}^{n} q\right)_{a, k}\right] \\
& +\frac{1}{2} \frac{\mathrm{d} G_{L}}{\mathrm{~d} \xi}\left(\widetilde{\xi}_{a, k}\right)\left[\left(\mathcal{I}_{R}^{n} q\right)_{a-1, k}-\left(\mathcal{I}_{L}^{n} q\right)_{a, k}\right]
\end{aligned}
$$

where

$$
\begin{aligned}
& \left(\hat{\mathcal{D}}_{i}^{n} q\right)_{a, k}= \\
& \begin{cases}\frac{1}{2}\left(\sum_{j=0}^{n_{\mathrm{vp}}-1} q_{a, j} \frac{\partial \phi_{a, j}}{\partial \xi} \widetilde{\xi}_{a, k}\right) & \\
\left.+\sum_{j=0}^{n_{\mathrm{vp}}-1} q_{a-1, j} \frac{\partial \phi_{a-1, j}}{\partial \xi}\left(\widetilde{\xi}_{a, k}\right)\right) & \text { if } k=0, \\
\sum_{j=0}^{n_{\mathrm{vp}}-1} q_{a, j} \frac{\partial \phi_{a, j}}{\partial \xi}\left(\widetilde{\xi}_{a, k}\right) & \text { if } 0<k<n \\
\frac{1}{2}\left(\sum_{j=0}^{n_{\mathrm{vp}}-1} q_{a, j} \frac{\partial \phi_{a, j}}{\partial \xi} \widetilde{\xi}_{a, k}\right)+ & \\
\left.\sum_{j=0}^{n_{\mathrm{v}}-1} q_{a+1, j} \frac{\partial \phi_{a+1, j}}{\partial \xi}\left(\widetilde{\xi}_{a, k}\right)\right) & \text { if } k=n_{\mathrm{vp}},\end{cases}
\end{aligned}
$$

and $G_{L}$ and $G_{R}$ are the local flux correction functions, which are chosen to satisfy

$$
\begin{aligned}
& G_{L}\left(\xi_{a, 0}\right)=1, G_{L}\left(\xi_{a, n_{\mathrm{vp}}-1}\right)=0, \\
& G_{R}\left(\xi_{a, 0}\right)=0, G_{R}\left(\xi_{a, n_{\mathrm{vp}}-1}\right)=1,
\end{aligned}
$$

and otherwise approximate zero throughout $\left[\xi_{a, 0}, \xi_{a, n_{\mathrm{vp}}-1}\right]$.

There is some flexibility in the discretization that depends on the specific choice of flux correction functions. Huynh (2007) gives a family of flux correction functions on the interval $[-1,1]$ denoted by $G_{k}$ for $k=1,2, \ldots$ In particular, we are interested in $G_{1}$ (the Radau polynomials) and $G_{2}$, which have the special property of $\mathrm{d} G_{2} / \mathrm{d} x=0$ at all Gauss-Lobatto points. Although either choice of flux correction function leads to a valid discretization for $n_{\mathrm{vp}}>1$, when $n_{\mathrm{vp}}=1$ a consistent differential operator is recovered only with $G_{2}$. Hence, for the remainder of this text we will adopt the flux correction function $G_{2}$. For this choice, the flux correction function satisfies

$\frac{\partial G_{2}}{\partial x}=\frac{\left(n_{\mathrm{vp}}+1\right)\left[P_{n_{\mathrm{vp}}+1}(x)-x P_{n_{\mathrm{vp}}}(x)\right]}{2(x-1)}$,

where $P_{N}(x)$ is the Legendre polynomial of order $N$. In the limit as $x$ approaches the boundaries of the reference element, a simplified expression emerges:

$$
\lim _{x \rightarrow+1} \frac{\partial G_{2}}{\partial x}=n_{\mathrm{vp}}\left(n_{\mathrm{vp}}+1\right) .
$$

On the interval $\left[\widetilde{\xi}_{j, 0}, \widetilde{\xi}_{j, n_{\mathrm{vp}}-1}\right]$ we have

$$
\begin{array}{r}
\frac{\partial G_{R}}{\partial \xi}(\xi)=\frac{1}{\Delta \xi_{a}} \frac{\partial G_{2}}{\partial x}\left[\frac{2\left(\xi-\xi_{j, 0}\right)}{\Delta \xi_{a}}-1\right] \\
\frac{\partial G_{L}}{\partial \xi}(\xi)=-\frac{1}{\Delta \xi_{a}} \frac{\partial G_{2}}{\partial x}\left[\frac{2\left(\xi_{j, n_{\mathrm{vp}-1}}-\xi\right)}{\Delta \xi_{a}}-1\right]
\end{array}
$$

\subsubsection{Second derivative operators in the vertical}

The second derivative operators are used in viscosity and hyperviscosity calculations. They are obtained as approximations to the equation

$\mathcal{L}(v) q \approx v \frac{\partial^{2} q}{\partial \xi^{2}}$,

subject to Neumann (no-flux) boundary condition

$\frac{\partial q}{\partial \xi}=0 \quad$ at $\xi=0$ and $\xi=1$.

For the viscous operator from interfaces to interfaces, the discretization is obtained from the variational (weak) formulation. Specifically, from Eq. (38) and integration by parts,

$$
\int_{0}^{1}\left(\mathcal{L}_{i}^{i} q\right)_{b, n} \widetilde{\phi}_{a, k} \mathrm{~d} \xi=\left.\frac{\partial q}{\partial \xi} \widetilde{\phi}_{a, k}\right|_{0} ^{1}-\int_{0}^{1} \frac{\partial q}{\partial \xi} \frac{\partial \widetilde{\phi}_{a, k}}{\partial \xi} \mathrm{d} \xi .
$$

Then using Eqs. (24), (39), and the assumption of orthogonality of basis functions $\widetilde{\phi}$ under quadrature,

$$
\left(\mathcal{L}_{i}^{i} q\right)_{a, k}=-\frac{1}{\int_{0}^{1} \widetilde{\phi}_{a, k}^{2} d \xi} \sum_{b=0}^{n_{\mathrm{ve}}-1} \sum_{n=0}^{n_{\mathrm{vp}}} \widetilde{q}_{b, n} \int_{0}^{1} \frac{\partial \widetilde{\phi}_{a, k}}{\partial \xi} \frac{\partial \widetilde{\phi}_{b, n}}{\partial \xi} \mathrm{d} \xi .
$$

For model interfaces on Gauss-Lobatto nodes, the integral is discretized via Gauss-Lobatto quadrature.

The viscous operator from levels to levels is derived in a similar manner, although the non-differentiability of $q$ at interfaces in the discontinuous basis means that we must rely on differentiation via Eq. (32). Consequently, the weak form

$$
\int_{\widetilde{\xi}_{a, 0}}^{\widetilde{\xi}_{a, v_{\mathrm{np}}}}\left(\mathcal{L}_{i}^{i} q\right)_{b, n} \phi_{a, k} d \xi=\left.\frac{\partial q}{\partial \xi} \phi_{a, k}\right|_{\widetilde{\xi}_{a, 0}} ^{\widetilde{\xi}_{a, v_{\mathrm{np}}}}-\int_{\widetilde{\xi}_{a, 0}}^{\widetilde{\xi}_{a, v_{\mathrm{np}}}} \frac{\partial q}{\partial \xi} \frac{\partial \phi_{a, k}}{\partial \xi} \mathrm{d} \xi .
$$

then leads to discrete operator

$$
\begin{aligned}
& \left(\mathcal{L}_{n}^{n} q\right)_{a, k}=\frac{1}{\int_{\widetilde{\xi}_{a, 0}}^{\widetilde{\xi}_{a, n \mathrm{vp}}} \phi_{a, k}^{2} \mathrm{~d} \xi} \\
& {\left[\left(\hat{\mathcal{L}}_{n}^{n} q\right)_{a, k}+\left(\mathcal{D}_{i}^{n} q\right)_{a, v_{\mathrm{np}}} \phi\left(\widetilde{\xi}_{a, v_{\mathrm{np}}}\right)-\left(\mathcal{D}_{i}^{n} q\right)_{a, 0} \phi\left(\widetilde{\xi}_{a, 0}\right)\right],}
\end{aligned}
$$

where

$$
\left(\hat{\mathcal{L}}_{n}^{n} q\right)_{a, k}=-\sum_{b=0}^{n_{\mathrm{ve}}-1} \sum_{n=0}^{n_{\mathrm{vp}-1}} q_{b, n} \int_{\widetilde{\xi}_{a, 0}}^{\widetilde{\xi}_{a, n_{\mathrm{vp}}}} \frac{\partial \phi_{a, k}}{\partial \xi} \frac{\partial \phi_{b, n}}{\partial \xi} \mathrm{d} \xi .
$$

For model levels on Gauss nodes, the integral is discretized directly via Gaussian quadrature. Note that the boundary condition implies that we must impose

$\left(\mathcal{D}_{i}^{n} q\right)_{0,0}=0 \quad$ and $\quad\left(\mathcal{D}_{i}^{n} q\right)_{v_{\mathrm{ne}}-1, v_{\mathrm{np}}}=0$. 


\subsubsection{Flow-dependent vertical hyperviscosity}

The basic spectral element method is an energy conservative scheme (Taylor and Fournier, 2010) that allows for the accumulation of energy at the shortest wavelengths. Following Ullrich (2014a) and Dennis et al. (2011), we impose explicit dissipation in the horizontal using a constant coefficient hyperviscosity. In the vertical, a constant coefficient hyperviscosity would have a rapid and adverse affect on hydrostatic balance in the absence of a hydrostatic reference state (Giraldo and Restelli, 2008). Consequently, in this paper we apply a localized hyperviscosity in the vertical column that is weighted by the contravariant vertical flow velocity $u^{\xi}$,

$\frac{\partial q}{\partial t}=\cdots+v_{z}\left|u^{\xi}\right| \frac{\partial^{2 k} q}{\partial \xi^{2 k}}$,

where $q \in\left\{u_{\alpha}, u_{\beta}, w, \theta, \rho\right\}$ and $k$ is a positive integer. The motivation for using $u^{\xi}$ stems from the observation that advective transport in the vertical occurs with speed $u^{\xi}$, and so this would be the corresponding wave speed that would enter into, for example, the Rusanov-Riemann solver in the context of discontinuous Galerkin or finite volume methods. In this sense, the flow-dependent hyperviscosity is a generalization of advective upwinding if applied simultaneously with the vertical advective operator. The Riemann solver interpretation also yields an appropriate estimate for the value of $v_{z}$,

$k=2: v_{z}=(1 / 2)(\overline{\Delta \xi})^{-1}$,

$k=4: v_{z}=-(1 / 12)(\overline{\Delta \xi})^{-3}$,

$k=6: v_{z}=(1 / 60)(\overline{\Delta \xi})^{-5}$,

where $\overline{\Delta \xi}=1 /\left(a n_{\mathrm{vp}}\right)$ is the average spacing between nodes in the vertical direction.

\subsubsection{The staggered nodal finite-element method}

The interpolation and differentiation operators given in the previous sections provide a framework for constructing staggered vertical grids in the context of the nonlinear system Eqs. (6)-(10). Furthermore, the SNFEM allows for discretizations of arbitrary order-of-accuracy via adjustments in $n_{\mathrm{vp}}$. For the present work, we investigate unstaggered (on interfaces), LOR ( $u, v, \rho, \theta$ on levels, $w$ on interfaces), and $\mathrm{CPH}(u, v, \rho$ on levels, $w, \theta$ on interfaces) configurations. The two key diagnosed variables, $\Pi$ and $u^{\xi}$ are collocated with $\rho$ and $w$, respectively. Table 1 provides a reference nomenclature for the various discrete derivative operators that arise in the SNFEM corresponding to the terms treated implicitly. In general, we will use subscripts and superscripts $i$ and $n$ denote quantities computed on "interfaces" or "levels", respectively. When needed, the contravariant $\alpha$ and $\xi$ velocity are computed via

$\left(u^{j}\right)=g^{j \alpha}\left(u_{\alpha}\right)+g^{j \beta}\left(u_{\beta}\right)+g^{j \xi}\left|\boldsymbol{g}_{\xi}\right| w$, where $j \in\{\alpha, \xi\}$ and all covariant velocities are first interpolated to levels or interfaces (wherever $u^{j}$ is needed) prior to evaluation.

For example, applying the discrete derivative operators with Lorenz staggering to Eqs. (6)-(10) and neglecting flow in the $\beta$ direction gives

$$
\begin{aligned}
& \frac{\partial\left(u_{\alpha}\right)_{n}}{\partial t}=-\mathcal{D}_{\alpha}\left(K_{n}+\Phi_{n}\right)-\theta_{n} \mathcal{D}_{\alpha}\left(\Pi_{n}\right)-\left(u^{\xi}\right)_{n} \\
& {\left[\mathcal{D}_{n}^{n} u_{\alpha}-\mathcal{D}_{\alpha} \mathcal{I}_{n}^{i}\left(u_{\xi}\right)_{i}\right],} \\
& \left(\frac{\partial r}{\partial \xi}\right)_{i} \frac{\partial w_{i}}{\partial t}=\underbrace{-\mathcal{D}_{i}^{n}\left(K_{n}+\Phi_{n}\right)-\mathcal{I}_{i}^{n} \theta_{n}\left(\mathcal{D}_{i}^{n} \Pi_{n}\right)} \\
& \underbrace{+\left(u^{\alpha}\right)_{i} \mathcal{D}_{n}^{i} u_{\alpha}}-\mathcal{I}_{i}^{n}\left[\left(u^{\alpha}\right)_{n} \mathcal{D}_{\alpha} \mathcal{I}_{n}^{i}\left(u_{\xi}\right)_{i}\right], \\
& \frac{\partial \theta_{n}}{\partial t}=-\left(u^{\alpha}\right)_{n}\left(\mathcal{D}_{\alpha} \theta_{n}\right) \underbrace{-\left[\mathcal{I}_{n}^{i}\left(u^{\xi}\right)_{i}\right]\left(\mathcal{D}_{n}^{n} \theta_{n}\right)}, \\
& \frac{\partial \rho_{n}}{\partial t}=-\frac{1}{J_{n}} \mathcal{D}_{\alpha}\left[J_{n} \rho_{n}\left(u^{\alpha}\right)_{n}\right] \\
& \underbrace{-\frac{1}{J_{n}} \mathcal{D}_{n}^{i}\left[J_{i}\left(\mathcal{I}_{i}^{n} \rho_{n}\right)\left(u^{\xi}\right)_{i}\right]} .
\end{aligned}
$$

Here the vertical interpolation operators are defined in Sect. 3.2.1, the derivative operators are defined in Sect. 3.2.2, and the horizontal derivative operator $\mathcal{D}_{\alpha}$ represents the standard collocated spectral element derivative operator.

It is important to note the great deal of flexibility available in the computation of spatial terms in Eqs. (49)-(52). In particular, covariant/contravariant velocity components (needed in the advection of $\theta$ ) and the specific kinetic energy $K$ may be composed with different interpolation sequences and preliminary experiments have suggested that stability of the method may depend on such variations, particularly in the presence of steep topography.

\subsection{Temporal discretization}

Many options are available for the temporal discretization of the semi-discrete equations, including several fully explicit and implicit-explicit schemes (Ascher et al., 1997). One simple temporal discretization is investigated here, which utilizes Strang splitting for the dynamics and operator splitting for the hyperviscosity. The Eqs. (6)-(10) are written in the form

$$
\frac{\partial \psi}{\partial t}-f(\boldsymbol{x}, \psi)=g(\boldsymbol{x}, \psi),
$$

where $f(\boldsymbol{x}, \psi)$ denotes terms associated with non-stiff modes, i.e., horizontally propagating modes and vertical advection of horizontal velocity. The function $g(\boldsymbol{x}, \psi)$ denotes geometrically stiff terms associated with all vertical derivatives except for vertical advection of horizontal velocity. The model follows the approach of Ullrich and Jablonowski (2012) by treating non-stiff terms using an explicit temporal 
operator and stiff terms using an implicit operator. For the current study, the terms with under-braces in Eqs. (49)-(52) are treated implicitly in order to avoid time step limitations due to vertically propagating sound waves.

For the first time step, an implicit update is applied,

$\psi^{(0)}=\psi^{n}+\frac{\Delta t}{2}\left(\mathcal{I}-\frac{\Delta t}{2} \mathcal{D} \mathcal{G}\left(\psi^{n}\right)\right)^{-1} \mathcal{G}\left(\psi^{n}\right)$,

where $\mathcal{G}\left(\psi^{n}\right)$ represents the discretization described in Sect. 3.2 and $\mathcal{D} \mathcal{G}\left(\psi^{n}\right)=\partial \mathcal{G} / \partial \psi^{n}$. For later time steps, the implicit update is instead obtained from a stored tendency,

$\psi^{(0)}=\psi^{n}+\frac{\Delta t}{2} \bar{\psi}$

Explicit terms are evolved using a Runge-Kutta method, which supports a large stability bound for spatial discretizations with purely imaginary eigenvalues. This particular scheme is based on Kinnmark and Gray (1984a, b) and takes the form

$$
\begin{aligned}
& \psi^{(1)}=\psi^{(0)}+\frac{\Delta t}{5} f\left(\psi^{(0)}\right) \\
& \psi^{(2)}=\psi^{(0)}+\frac{\Delta t}{5} f\left(\psi^{(1)}\right) \\
& \psi^{(3)}=\psi^{(0)}+\frac{\Delta t}{3} f\left(\psi^{(2)}\right) \\
& \psi^{(4)}=\psi^{(0)}+\frac{2 \Delta t}{3} f\left(\psi^{(3)}\right) \\
& \psi^{(5)}=-\frac{1}{4} \psi^{(0)}+\frac{5}{4} \psi^{(1)}+\frac{3 \Delta t}{4} f\left(\psi^{(4)}\right) .
\end{aligned}
$$

Hyperviscosity is then applied in accordance with Ullrich (2014a), with scalar hyperviscosity used for all scalar quantities and vector hyperviscosity used for the horizontal velocity field. Mathematically, the update takes the form,

$$
\begin{aligned}
& \psi_{\mathrm{s}}^{(6)}=\psi_{\mathrm{s}}^{(5)}+\Delta t \mathcal{H}(v) \mathcal{H}(1) \psi_{\mathrm{s}}^{(5)} \\
& \boldsymbol{u}^{(6)}=\boldsymbol{u}^{(5)}+\Delta t \mathcal{H}\left(v_{d}, v_{v}\right) \mathcal{H}(1,1) \boldsymbol{u}^{(5)}
\end{aligned}
$$

where $\psi_{\mathrm{s}} \in\{\theta, w, \rho\}$.

When active, Rayleigh friction is applied via backward Euler to relax all prognostic variables to a specified reference state,

$$
\psi^{(7)}=\gamma \psi^{(6)}+(1-\gamma) \psi^{\mathrm{ref}}
$$

where $\gamma=\left[1+v_{r}(\boldsymbol{x}) \Delta t\right]^{-1}$ is in terms of the Rayleigh friction strength $v_{r}(\boldsymbol{x})$.

In accordance with Strang splitting, a final implicit update is applied,

$$
\begin{aligned}
\bar{\psi} & =\left(\mathcal{I}-\frac{\Delta t}{2} \mathcal{D} \mathcal{G}\left(\psi^{(7)}\right)\right)^{-1} \mathcal{G}\left(\psi^{(7)}\right), \\
\psi^{n+1} & =\psi^{(7)}+\frac{\Delta t}{2} \bar{\psi} .
\end{aligned}
$$

\section{Validation}

In this section we present a set of test cases with the purpose of investigating the performance of the SNFEM for mesoscale atmospheric modeling. Our emphasis is on a wide range of resolutions from the global scale $(200 \mathrm{~km})$ to the large-eddy scale $(5 \mathrm{~m})$. These scales transition from hydrostatic to scales where all nonlinear terms in the Eqs. (6)-(10) become significant. For our experiments we will hold the following components of the computations constant:

1. The horizontal discretization is kept as a standard fourth-order spectral element formulation for all simulations, as outlined in Sect. 3.1.

2. The time integration scheme is based on Strang-split IMplicit EXplicit (IMEX) outlined in Sect. 3.3.

3. Vertical terms $\frac{\partial}{\partial z}$ are integrated implicitly using the generalized minimal residual method (GMRES) with no preconditioner. Efforts are underway to determine the most efficient preconditioner for this system. We have also implemented an analytical Jacobian for the vertical solve, which appears to be the most computationally efficient option.

4. Reference solutions employ consistent fourth-order vertical and horizontal discretizations at a resolution at least twice as fine as experiments

5. The total number of vertical levels in each test is kept constant. Only the vertical order of accuracy is changed and consequently the distribution of grid spacing according to the locations of element nodes.

For these tests, we investigate the effect of a relatively high-order $n_{\mathrm{vp}}=10$ vertical coordinate on flow results at resolutions coarser than the reference solutions. Our hypothesis is that flow structures and measures of interest will be better approximated using the high-order discretization. We consider the properties of our arbitrary order methods in the context of meshes with mixed grid resolutions such as static and adaptive variable resolution experiments. A primary benefit of using the higher-order SNFEM is improved accuracy even with a coarser vertical grid.

Reference results are computed with a consistent spatial (horizontal and vertical) discretization or order "O4". Experiments done at coarser resolutions with varying vertical order of accuracy are titled "VO(no.)".

\subsection{Steady-state geostrophically balanced flow in a channel}

The first test represents steady-state geostrophically balanced flow in a channel and is based on a new test case defined by Ullrich et al. (2015). The domain is a channel of dimensions $L_{x} \times L_{y} \times L_{z}$ with periodic boundaries in the $x$ direction and no-flux conditions at all other interfaces. In this case 

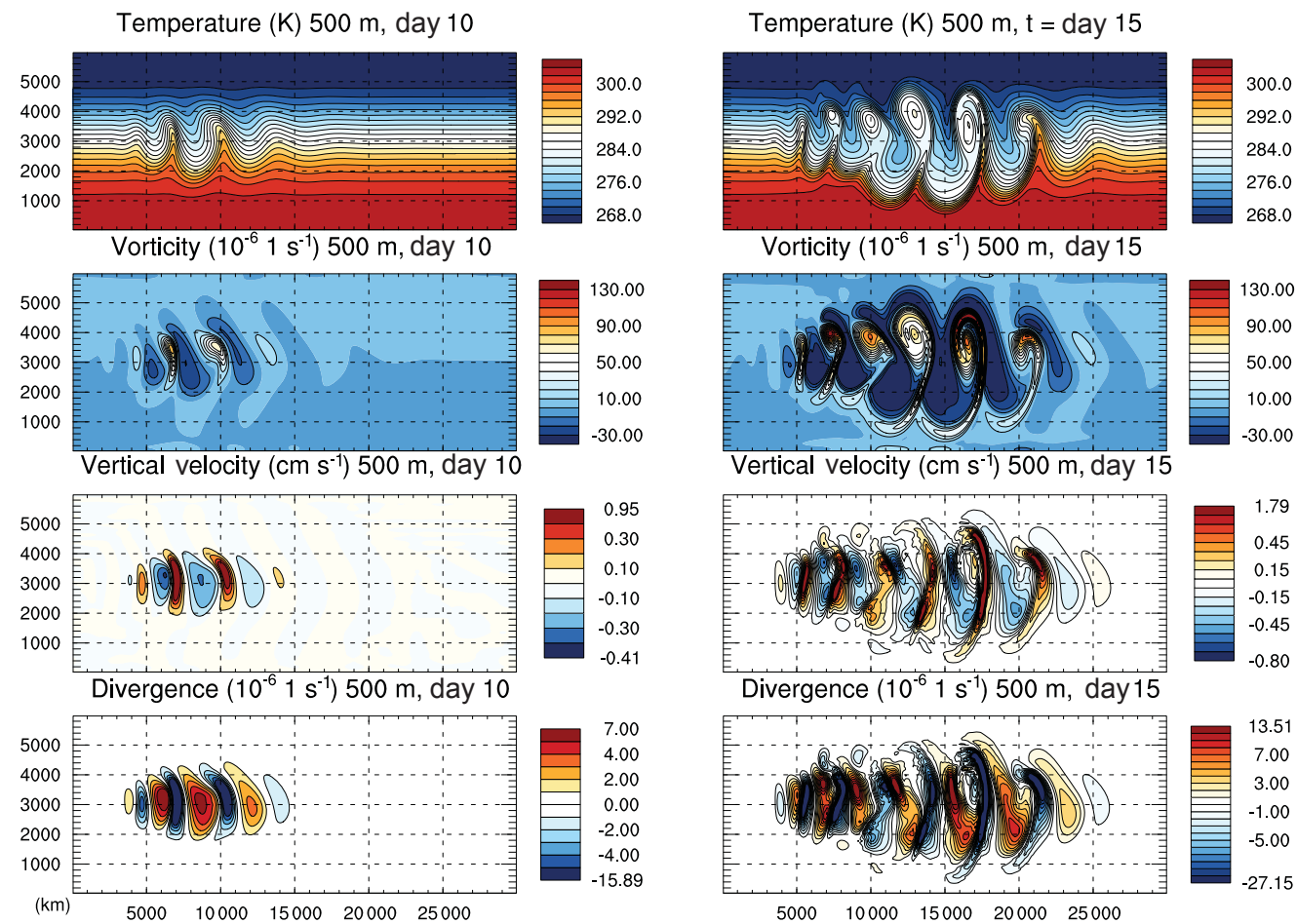

Figure 3. Baroclinic wave in a 3-D Cartesian channel at the reference resolution $\Delta x=100 \mathrm{~km}, \Delta y=100 \mathrm{~km}, \Delta z=1 \mathrm{~km}$ at vertical fourthorder accuracy (VO4). From top to bottom, temperature, vorticity, vertical velocity, and divergence are shown at day 10 (left) and 15 (right) and at an elevation of $500 \mathrm{~m}$. Contour intervals: temperature $2 \mathrm{~K}$, vorticity $1.0 \times 10^{-5} \mathrm{~s}^{-1}$, divergence $5.0 \times 10^{-6} \mathrm{~s}^{-1}$, and vertical velocity $2.0 \times 10^{-3} \mathrm{~m} \mathrm{~s}^{-1}$.

we choose $L_{x}=30000 \mathrm{~km}, L_{y}=6000 \mathrm{~km}$, and $L_{z}=30 \mathrm{~km}$. The shorter zonal width compared with that of Ullrich et al. (2015) was chosen for reasons of computational efficiency and did not affect the final solution. The initial flow is comprised of a zonally symmetric mid-latitudinal jet, defined so that the wind is zero at the surface and along the $y$ boundary. Hyperviscosity is applied in the horizontal and vertical at fourth order as well as a Rayleigh layer at the top and longitudinal boundaries. The Rayleigh layers are used to prevent the accumulation of standing wave reflections in the flow. This formulation can either be on an $f$ plane or $\beta$ plane, which have Coriolis parameters

$f=f_{0}, \quad$ and $\quad \beta=f_{0}+\beta_{0}\left(y-y_{0}\right)$,

respectively, where $f_{0}=2 \Omega \sin \varphi_{0}$ and $\beta_{0}=2 a^{-1} \Omega \cos \varphi_{0}$ at latitude $\varphi_{0}=45^{\circ} \mathrm{N}$. Here, the radius of the Earth is $a=6371.229 \times 10^{3} \mathrm{~m}$, its angular velocity is $\Omega=7.292 \times$ $10^{-5} \mathrm{~s}^{-1}$ and $y_{0}=L_{y} / 2$ is the center point of the domain in the $y$ direction.

The simulation is performed for the original $\beta$-plane configuration outlined in Ullrich et al. (2015) where the jet is perturbed directly by a "bump" in the zonal wind that is vertically uniform where $u_{p}=1.0 \mathrm{~m} \mathrm{~s}^{-1}$ centered at $x_{c}=$ $2000 \mathrm{~km}$ and $y_{c}=2500 \mathrm{~km}$. $u^{\prime}(x, y)=u_{p} \exp \left[-\left(\frac{\left(x-x_{c}\right)^{2}+\left(y-y_{c}\right)^{2}}{L_{p}^{2}}\right)\right]$

The grid spacing for the reference solution is $\Delta x=50 \mathrm{~km}$, $\Delta y=50 \mathrm{~km}, \Delta z=0.75 \mathrm{~km}$, and $\Delta t=30 \mathrm{~s}$. Experiments are conducted at vertical order 2,4 , and 10 at a resolution of $\Delta x=200 \mathrm{~km}, \Delta y=200 \mathrm{~km}, \Delta z=1.5 \mathrm{~km}$, and $\Delta t=240 \mathrm{~s}$. The fourth-order scalar and vector (vorticity and divergence separately) diffusion coefficients are given by

$$
\begin{aligned}
v_{\text {scalar }} & =1.0 \times 10^{14}\left(\frac{\Delta x}{L_{\text {ref }}}\right)^{3.2} \mathrm{~m}^{4} \mathrm{~s}^{-1}, \\
v_{\text {vorticity }} & =1.0 \times 10^{14}\left(\frac{\Delta x}{L_{\text {ref }}}\right)^{3.2} \mathrm{~m}^{4} \mathrm{~s}^{-1}, \\
v_{\text {divergence }} & =1.0 \times 10^{14}\left(\frac{\Delta x}{L_{\text {ref }}}\right)^{3.2} \mathrm{~m}^{4} \mathrm{~s}^{-1},
\end{aligned}
$$

where $\Delta x$ is the element length in the $x$ direction and $L_{\text {ref }}=$ $11.0 \times 10^{5} \mathrm{~m}$ is the reference length used for this test case. For this test, vertical flow-dependent viscosity is disabled since it did not have a clear impact on the solution.

The baroclinic instability is a primary mechanism for the development of mid-latitude storm systems and so it is important that an atmospheric modeling platform reproduce 

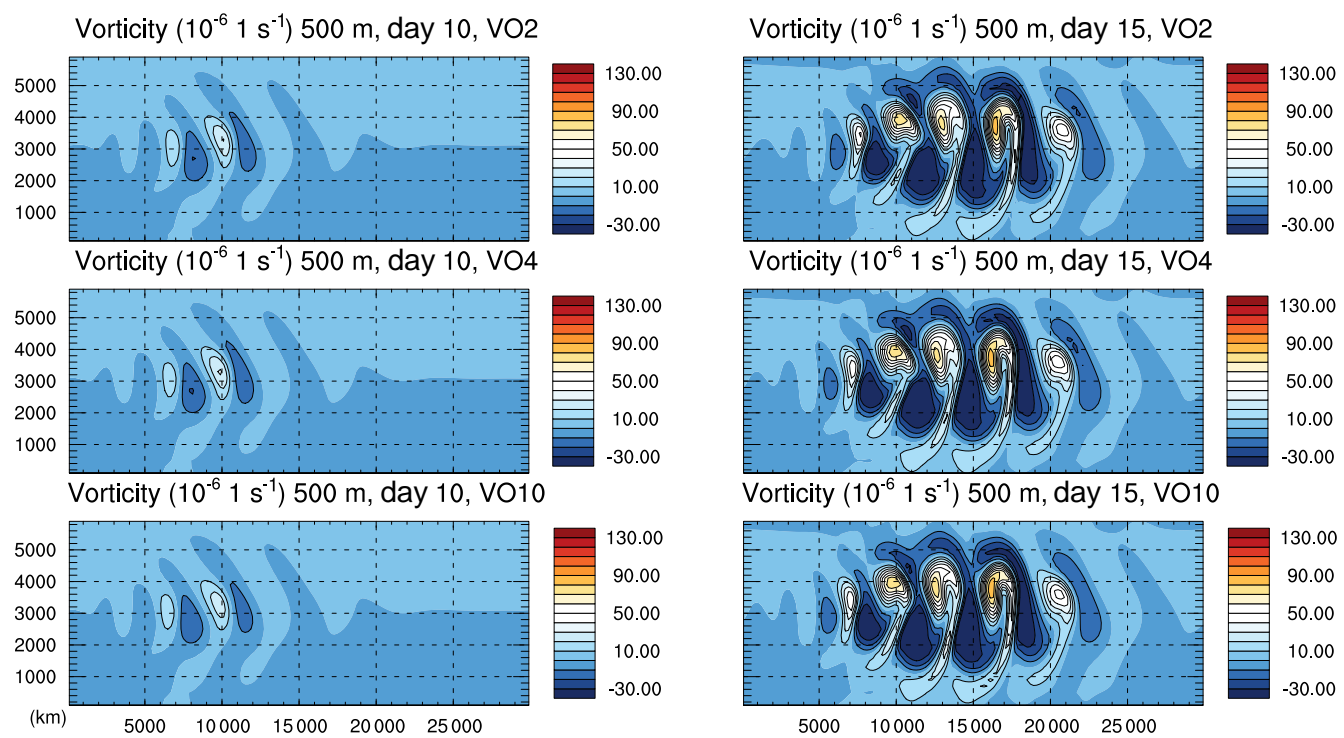

Figure 4. Baroclinic wave in a Cartesian channel at vertical orders 2, 4, and 10 . Vorticity at $500 \mathrm{~m}$ on days 10 and 15 at the resolution $\Delta x=200 \mathrm{~km}, \Delta y=200 \mathrm{~km}, \Delta z=1.5 \mathrm{~km}$. Contour interval: $1.0 \times 10^{-5} \mathrm{~s}^{-1}$.

these phenomena accurately. We present a reference solution of the baroclinic wave shown in Fig. 3 that is approaching the transition into the non-hydrostatic regime. We are interested in estimates of vertical motion where the reference solution shows maxima on the order of $2 \mathrm{~cm} \mathrm{~s}^{-1}$. Regions of strong vertical motion correspond to strong horizontal gradients in the vorticity and temperature fields and we expect that nonhydrostatic effects will be locally significant.

The reference solution for temperature and vorticity at $500 \mathrm{~m}$ elevation shown here can be compared at day 10 with the original results from Ullrich et al. (2015) produced with MCore Ullrich and Jablonowski (2012) to verify that Tempest is computing a consistent solution. In particular we expect that vertical motion will be under-predicted in coarser models at a given order of accuracy.

The vorticity field at coarse resolution (Fig. 4) is largely unaffected by changes in vertical order. However, the vertical velocity (Fig. 5), and by association the horizontal divergence (not shown), shows a substantial increase in magnitude as order increases. This increase aligns the vertical velocity more closely with the reference solution magnitude (greater than $1 \mathrm{~cm} \mathrm{~s}^{-1}$ ) using the 10th-order vertical coordinate as shown in Fig. 5. We conclude that although the higher-order vertical coordinate does not substantially impact the horizontal character of the solution, it does better capture the magnitude of vertical velocity, particularly in frontal regions. We note that the coarse resolution chosen here is nearly double that of current operational climate modeling systems and well within the hydrostatic regime.

\subsection{Schär mountain}

Atmospheric flows are strongly influenced by the lower boundary, where topographically induced waves transport momentum and energy vertically. Schär et al. (2002) described a uniform zonal flow field over orography that leads to the generation of a stationary mountain response, consisting of a linear combination of hydrostatic and nonhydrostatic waves. The atmosphere is initially under uniform stratification with constant Brunt-Väisälä frequency $\mathcal{N}=$ $0.01 \mathrm{~s}^{-1}$. The temperature and pressure are $p_{0}=1000 \mathrm{hPa}$ and $T_{0}=280 \mathrm{~K}$ at $z=0 \mathrm{~m}$. To trigger the standing waves, an initial uniform mean flow of $\bar{u}=10 \mathrm{~m} \mathrm{~s}^{-1}$ is prescribed over the topographic profile given by

$h_{T}(x)=h_{c} \exp \left[-\left(\frac{x}{a_{c}}\right)^{2}\right] \cos ^{2}\left(\frac{\pi x}{\lambda}\right)$,

with parameters $h_{c}=250 \mathrm{~m}, \lambda=4000 \mathrm{~m}$, and $a_{c}=5000 \mathrm{~m}$. The simulation domain is $(x, z) \in[-30,30] \times[0,25] \mathrm{km}$ with a no-flux boundary specified along the bottom surface. Freeflow boundary conditions are prescribed at the top and lateral boundaries with a Rayleigh layer $10 \mathrm{~km}$ wide along the lateral boundaries and $10 \mathrm{~km}$ deep at the model top. Note that the domain bounds differ from Schär et al. (2002) to minimize the effect of the Rayleigh layers on the flow interior. Also, the Rayleigh layer is applied to progressively and smoothly increase in strength up to the boundaries. The simulation is run to $t=10 \mathrm{~h}$, when the solution has reached a quasi-steady state. For these simulations, no explicit dissipation is applied in either the horizontal or vertical and Coriolis forcing is set to zero throughout. 


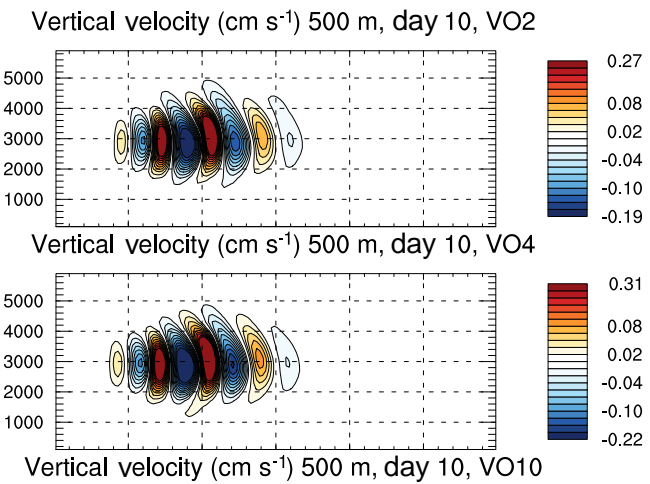

Vertical velocity $\left(\mathrm{cm} \mathrm{s}^{-1}\right) 500 \mathrm{~m}$, day 15, VO2

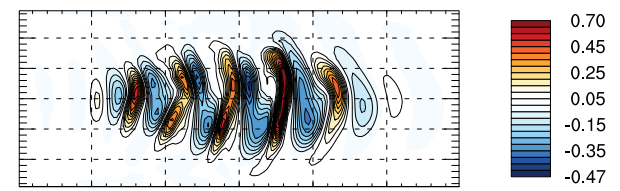

Vertical velocity $\left(\mathrm{cm} \mathrm{s}^{-1}\right) 500 \mathrm{~m}$, day 15 , VO4

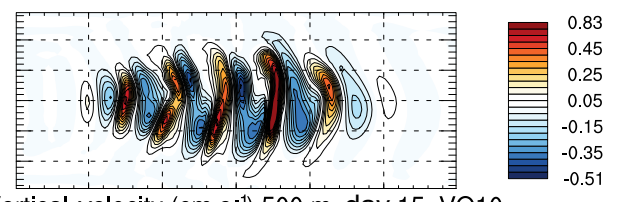

Vertical velocity $\left(\mathrm{cm} \mathrm{s}^{-1}\right) 500 \mathrm{~m}$, day 15 , VO10
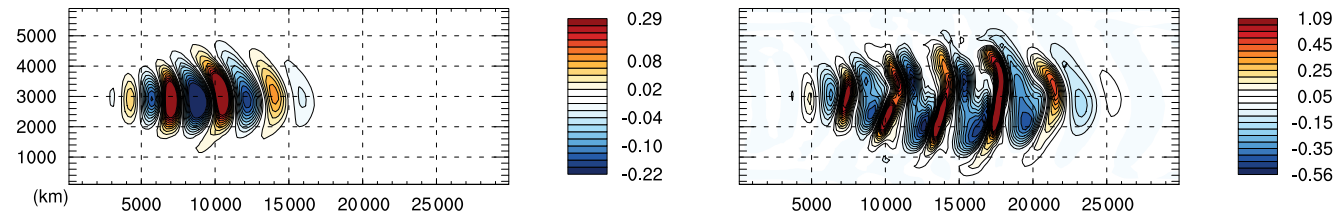

Figure 5. Baroclinic wave in a Cartesian channel at vertical orders 2, 4, and 10 . Vertical velocity at $500 \mathrm{~m}$ on days 10 and 15 at the resolution $\Delta x=200 \mathrm{~km}, \Delta y=200 \mathrm{~km}, \Delta z=1.5 \mathrm{~km}$. Contour interval: $2.0 \times 10^{-3} \mathrm{~m} \mathrm{~s}^{-1}$.
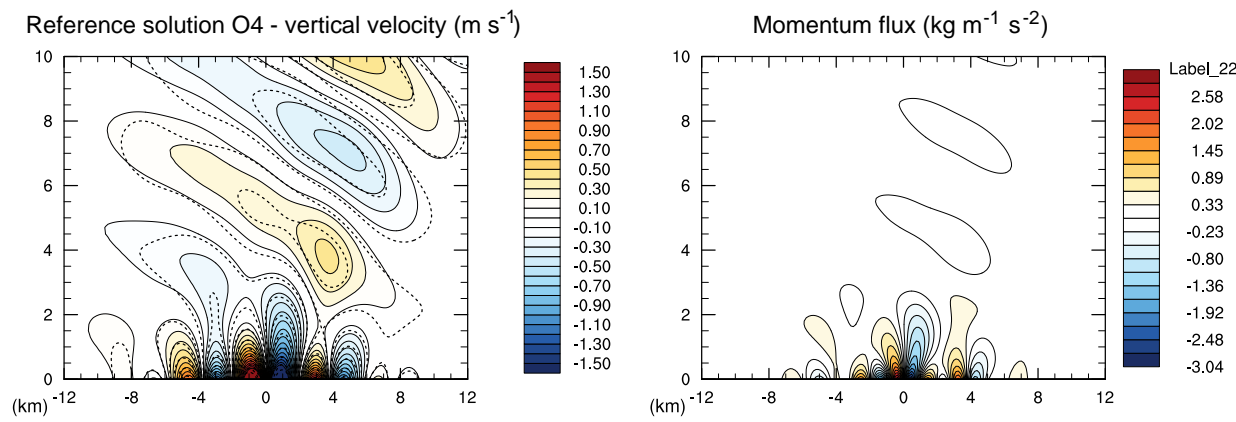

Figure 6. Schär flow at steady-state $(10 \mathrm{~h})$ vertical velocity in $\left(\mathrm{ms}^{-1}\right)$ at VO4. Reference resolution shown compared to the analytical solution (dotted contours) from linear mountain wave theory. $\Delta x=100 \mathrm{~m}$ and $\Delta z=100 \mathrm{~m}$. Contour interval: $0.1 \mathrm{~m} \mathrm{~s}^{-1}$.

To validate that Tempest produces the correct mountain wave response, the Schär mountain test was performed until $t=10 \mathrm{~h}$ with a relatively fine resolution of $\Delta x=100 \mathrm{~m}$, $\Delta z=100 \mathrm{~m}$ and $\Delta t=0.2 \mathrm{~s}$. As shown in Fig. 6 (left) Tempest accurately reproduces the vertical velocity field at the reference resolution (for comparison with another numerically derived solution, see Giraldo and Restelli, 2008). We also show the analytical solution based on linear mountain theory following Klemp et al. (2003) and Smith (1979) overlaid in dotted contours. As pointed out by Klemp et al. (2003), an inconsistent treatment of the topographic metric terms in this formulation can lead to the generation of spurious waves, which is not observed in this case.

As discussed in Thuburn and Woollings (2005) and Thuburn (2006), staggering is necessary to eliminate stationary computational modes that arise in collocated discretizations. To better understand the impact of staggering, Fig. 7 demonstrates the use of the collocated or unstaggered configuration, which shows a highly oscillatory stationary mode that pollutes the solution relative to the Lorenz configuration at the same resolution. The plots show errors in the vertical velocity near the bottom boundary condition and errors throughout the flow field due to the vertical mode. This artifact is conspicuously absent from both LOR and CPH runs.

Because our model makes use of a terrain-following coordinate, it is expected that a hydrostatically balanced rest state is not exactly preserved over topography. Imbalance will arise as a consequence of inexact cancellation of the terrain-following and vertical pressure gradient terms in the discrete equations. Experiments carried out with zero background flow in the presence of topographic features shown in Fig. 8 indicate that errors in vertical velocity are dominated by the horizontal discretization. We note that improvements with vertical order of accuracy are apparent when going from second order to fourth order, but differences are small at higher orders of accuracy. These errors can be removed completely with a vertical reference state (Giraldo and Restelli, 

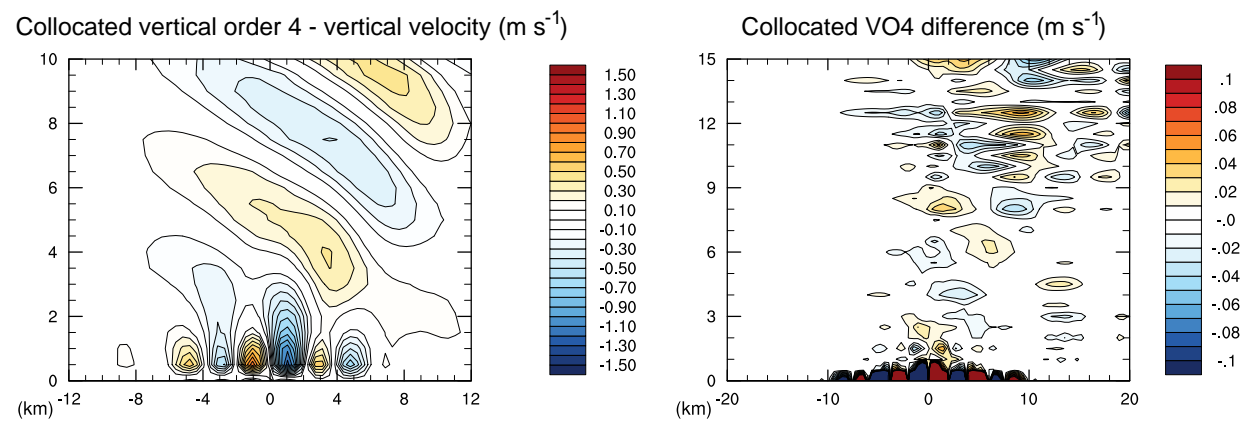

Figure 7. Schär flow at steady state $(10 \mathrm{~h})$. Collocated method (all variables on column levels) results compared to staggered (Lorenz) solution at the same spatial order and resolution. $\Delta x=200 \mathrm{~m}$ and $\Delta z=200 \mathrm{~m}$. Contour intervals: vertical velocity $0.1 \mathrm{~m} \mathrm{~s}{ }^{-1}$ and vertical velocity difference versus reference $0.0125 \mathrm{~m} \mathrm{~s}^{-1}$.

2008), but such a state is difficult to utilize for global simulations and so may not be desirable in practice.

Experiments are conducted at vertical order 2, 4, 10, and 40 (in the limit where the polynomial order is equal to the total number of levels, denoted ST) at a relatively coarse resolution of $\Delta x=500 \mathrm{~m}, \Delta z=500 \mathrm{~m}$, and $\Delta t=0.4 \mathrm{~s}$. Results are depicted in Fig. 9 and the difference against the reference solution in Fig. 10. The second-order results show substantial disagreement with the reference solution that is enhanced at altitude. This result appears to be associated with an overestimation of the vertical wavelength of the mountain response that arises from the lower-order discretization. At fourth order the upper atmosphere does not show substantial errors, and most differences are instead constrained to the near surface. These near-surface errors generally show consistent improvement as the vertical order-of-accuracy is increased. The discrepancy that appears at the highest peak of the Schär mountain $(x=0)$ is associated with slight differences in resolving the topography at coarser horizontal resolution than the reference solution.

We further compare the resulting profiles of momentum flux for all experiments in the Lorenz configuration (Fig. 11). We observe that the flux profile for the 2nd-order method has the greatest error, as expected from dispersion errors typical of low-order centered schemes (particularly in the upper atmosphere and near the surface). The higher-order methods show improvements in the structure and magnitude of the profiles (especially at the near-surface, when compared to the reference profile in black), but again appear to be influenced by the lower-order horizontal discretization. Furthermore, the results are strongly influenced by the Rayleigh layer showing a pronounced deviation in the flux profiles throughout the domain. The Rayleigh layer approximation to a free-flow boundary condition clearly introduces deficiencies that are exacerbated in the flux provided.

\subsection{Straka density current}

The density current test case of Straka et al. (1993) considered a cold bubble that sinks and spreads across the bottom boundary, driving the development of Kelvin-Helmholtz rotors. The original experiments by Straka et al. (1993) sought a converged solution through the use of second-order uniform diffusion applied to all prognostic variables. A value of $v=75 \mathrm{~m}^{2} \mathrm{~s}^{-1}$ was chosen so that a horizontal resolution of $\Delta x=25 \mathrm{~m}$ was sufficient for convergence. No-flux conditions are applied on all boundaries and the Coriolis forcing is set to zero.

The initial state consists of a hydrostatically balanced state with a uniform potential temperature of $\theta=300 \mathrm{~K}$. A standard pressure of $p_{0}=1000 \mathrm{hPa}$ is assumed. The cold bubble perturbation is applied to the $\theta$ field and is given by

$\theta^{\prime}= \begin{cases}0 & \text { if } r>1.0, \\ -\frac{\theta_{c}}{2}[1+\cos (\pi r)] & \text { if } r \leq 1.0,\end{cases}$

where $\theta_{c}=-15 \mathrm{~K}$ and

$r=\sqrt{\left(\frac{x-x_{c}}{x_{r}}\right)^{2}+\left(\frac{z-z_{c}}{z_{r}}\right)^{2}}$.

The domain is an enclosed box $(x, z) \in$ $[-25600,25600] \times[0,6400] \mathrm{m}$ with $t \in[0,900] \mathrm{s}$. The cold bubble is initially located at $\left(x_{c}, z_{c}\right)=(0,3000) \mathrm{m}$ with radius $\left(x_{r}, z_{r}\right)=(4000,2000) \mathrm{m}$. 


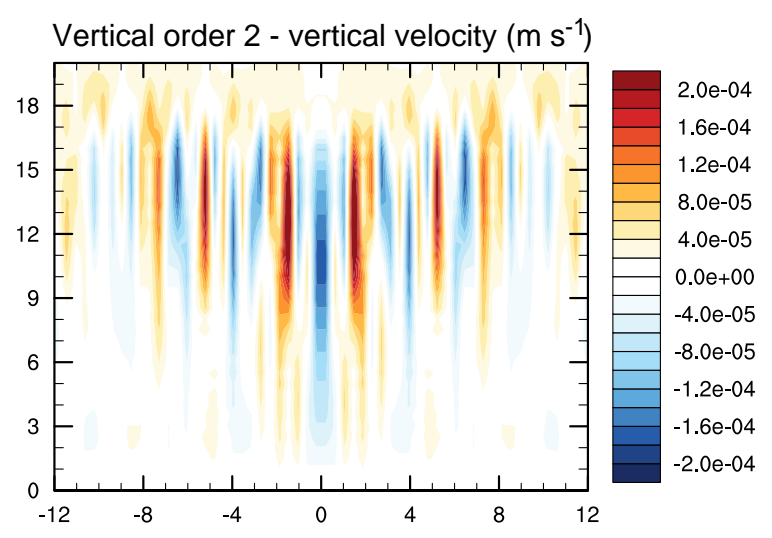

Vertical order 4 - vertical velocity $\left(\mathrm{m} \mathrm{s}^{-1}\right)$
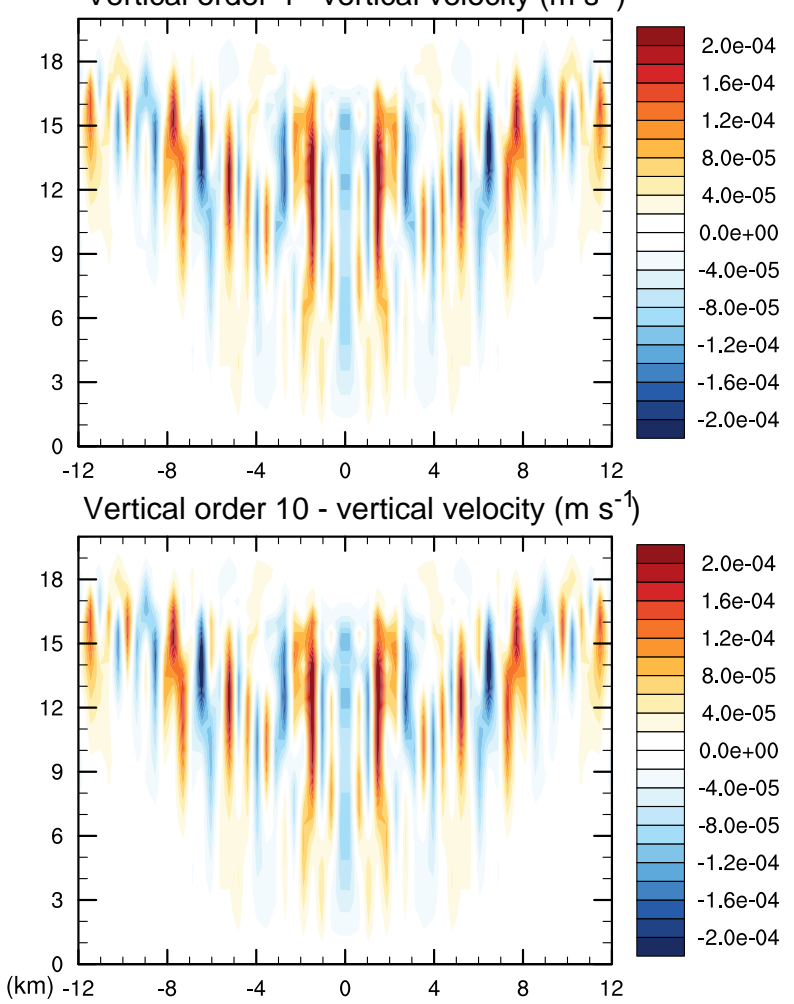

Figure 8. Still atmosphere experiment over Schär mountain profile at vertical orders 2,4 , and 10 showing errors in vertical velocity. $\Delta x=500 \mathrm{~m}$ and $\Delta z=500 \mathrm{~m}$. Contour interval: $2.0 \times 10^{-5} \mathrm{~m} \mathrm{~s}^{-1}$.

The fourth-order horizontal hyperdiffusion coefficients for all fields are given by

$$
\begin{aligned}
v_{\text {scalar }} & =5.0 \times 10^{12}\left(\frac{\Delta x}{L_{\text {ref }}}\right)^{3.2} \mathrm{~m}^{4} \mathrm{~s}^{-1}, \\
v_{\text {vorticity }} & =2.0 \times 10^{14}\left(\frac{\Delta x}{L_{\text {ref }}}\right)^{3.2} \mathrm{~m}^{4} \mathrm{~s}^{-1}, \\
v_{\text {divergence }} & =2.0 \times 10^{14}\left(\frac{\Delta x}{L_{\text {ref }}}\right)^{3.2} \mathrm{~m}^{4} \mathrm{~s}^{-1},
\end{aligned}
$$

where $\Delta x$ is the element length in the $x$ direction and $L_{\text {ref }}=$ $51200.0 \mathrm{~m}$ is the reference length used for this test case.
For the experiments with vertical flow-dependent hyperviscosity, the viscous coefficients are given by Eq. (47). The uniform Laplacian diffusion requires further stabilization via the addition of fourth-order scalar hyperviscosity in the horizontal and fourth-order vertical flow-dependent diffusion on all variables. This added diffusivity is necessary to control a horizontal stationary mode in the scalar fields and fast moving vertical modes that are a consequence of sound waves accumulating energy at the grid scale. However, the highly scale-selective nature of the high-degree operators does not significantly affect the structure of the reference solution as shown in Fig. 12.

The grid spacing for the reference solution is $\Delta x=25 \mathrm{~m}$ and $\Delta z=25 \mathrm{~m}$ with $\Delta t=0.01 \mathrm{~s}$. Experiments are further conducted at vertical order 2 and 10 at a resolution of $\Delta x=$ $200 \mathrm{~m}$ and $\Delta z=200 \mathrm{~m}$ with $\Delta t=0.01 \mathrm{~s}$.

For the density current, we emphasize results from the Lorenz (LOR) staggering. Under CPH staggering, the vertical advection term for potential temperature (see Table 1) on the bottom-most and top-most interfaces is exactly zero within our formulation. Consequently, within our formulation there is no mechanism to transport $\theta$ vertically from these interfaces leading to the development of a discontinuity in $\theta$ along the lower boundary. These gradients then enhance vertical heat fluxes above the surface, slowing the propagating cold pool as momentum is transported vertically. This inconsistency is counteracted by the application of uniform diffusion, which provides a mechanism by which $\theta$ can be exchanged with the bottom interface. However, flow-dependent vertical diffusion, which is weighted by $\left|u^{\xi}\right|$, does not permit exchange with the interface and so leads to inconsistency between the LOR and $\mathrm{CPH}$ staggering. In Fig. 12, the $\mathrm{CPH}$ staggering with flow-dependent diffusion leads to a relatively slow density current that is more convective near the boundary. Nonetheless, a better choice of flow-dependent coefficient could be made to mitigate this issue. Note that this issue with $\mathrm{CPH}$ can be counteracted by rewriting the vertical advection term as

$u^{\xi} \frac{\partial \theta}{\partial \xi}=\frac{\partial}{\partial \xi}\left(u^{\xi} \theta\right)-\theta \frac{\partial u^{\xi}}{\partial \xi}$,

although this form tends to be more unstable in practice

We often desire diffusion to be as weak as possible while still preserving the stability of the underlying method. However, as can be seen here, the structure of the density current is also strongly dependent on the dissipation mechanisms employed in the simulation. Here we present the reference solution equivalent to Straka et al. (1993) at the converged resolution. We also compare solutions with different diffusion mechanisms in Fig. 12 with corresponding cross sections in Figs. 14. The $1200 \mathrm{~m}$ cross sections indicate that experimental coarse resolutions are not converged in the case of reference uniform damping. In Table 2 it is apparent the reference solutions are sensitive to diffusion and differ significantly in structure, but the wave front positions compare with 

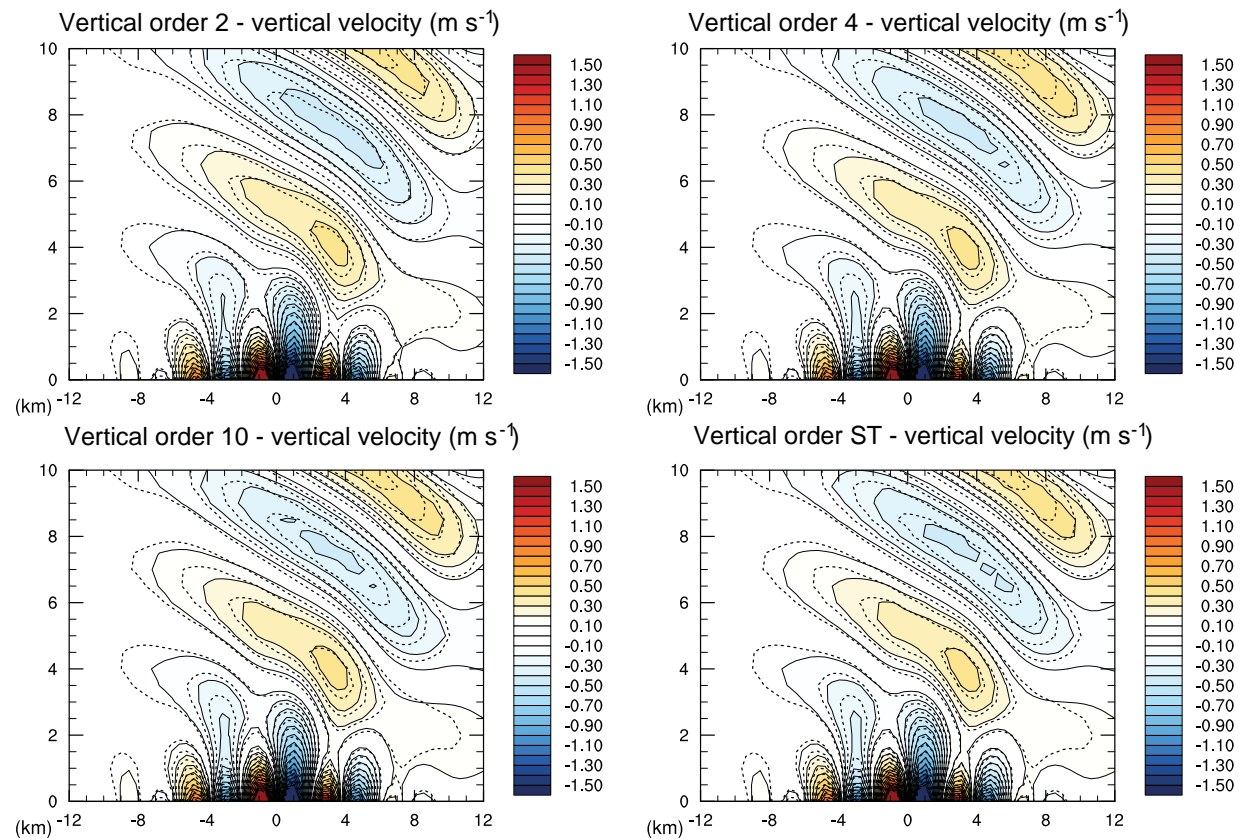

Figure 9. Schär flow at steady-state $(10 \mathrm{~h})$ vertical velocity in $\left(\mathrm{m} \mathrm{s}^{-1}\right)$ at various vertical orders of accuracy $(2,4,10$, and ST) where "ST" stands for single column element spectral transform $\left(n_{\mathrm{ve}}=1\right)$ with Lorenz (LOR) vertical staggering. Colored contours from Tempest compared to dotted contours for the analytical solution. $\Delta x=500 \mathrm{~m}$ and $\Delta z=500 \mathrm{~m}$. Contour interval: $0.1 \mathrm{~m} \mathrm{~s}^{-1}$.
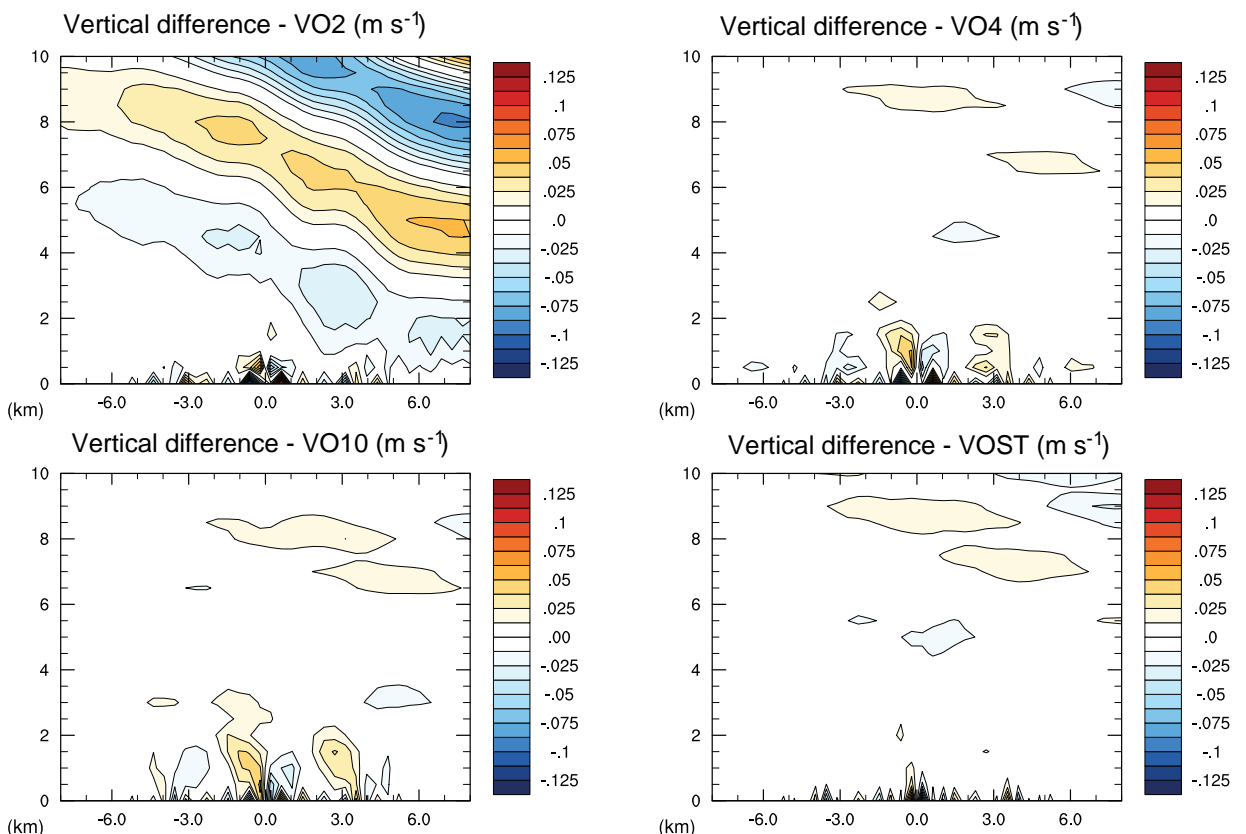

Figure 10. Schär flow steady state $(10 \mathrm{~h})$. Vertical velocity difference with respect to the reference solution (Fig. 6, left). Results are interpolated to a regular $z$ coordinate with $\Delta z=500 \mathrm{~m}$ in experiments and reference solution for differencing. Computations performed at $\Delta x=500 \mathrm{~m}$ and $\Delta z=500 \mathrm{~m}$. Contour interval: $0.0125 \mathrm{~m} \mathrm{~s}^{-1}$.

good precision to the solution given by Straka et al. (1993). This would indicate that momentum fluxes are comparable, but close inspection of the eddy structure suggests significant differences exist throughout, as noted above, and with the appearance of detached eddies when the high-order flowdependent viscosity is used exclusively.

From Table 2 it is apparent our coarse-resolution experimental solutions are slow with reference damping and 
Table 2. Cold wave front position $\left(\theta^{\prime}=-1.0 \mathrm{~K}\right)$ for all orders of accuracy and diffusion methods. Reference damping is uniform second-order diffusion on all prognostic variables such that $v=75 \mathrm{~m}^{2} \mathrm{~s}^{-1}$ combined with horizontal hyperdiffusion on scalars and vertical fourth-order up-wind diffusion. The reference solution wave front position (finite difference (FD) method at $25 \mathrm{~m}$ resolution) by Straka et al. (1993) is shown in bold (REFC) compared to the equivalent result from Tempest.

\begin{tabular}{lllr}
\hline Method-stagger & Vertical order @ resolution & Diffusion method & Wave front $(\mathrm{km})$ \\
\hline SNFEM-LOR & $2 @ \Delta x=190 \mathrm{~m}$ & Reference damping & 14.21 \\
SNFEM-LOR & $2 @ \Delta x=190 \mathrm{~m}$ & Up-wind order 2 & 14.59 \\
SNFEM-LOR & $2 @ \Delta x=190 \mathrm{~m}$ & Up-wind order 4 & 15.68 \\
SNFEM-LOR & $4 @ \Delta x=190 \mathrm{~m}$ & Reference damping & 14.18 \\
SNFEM-LOR & $4 @ \Delta x=190 \mathrm{~m}$ & Up-wind order 2 & 14.58 \\
SNFEM-LOR & $4 @ \Delta x=190 \mathrm{~m}$ & Up-wind order 4 & 15.47 \\
SNFEM-LOR & $10 @ \Delta x=190 \mathrm{~m}$ & Reference damping & 14.22 \\
SNFEM-LOR & $10 @ \Delta x=190 \mathrm{~m}$ & Up-wind order 2 & 14.61 \\
SNFEM-LOR & $10 @ \Delta x=190 \mathrm{~m}$ & Up-wind order 4 & 15.33 \\
\hline FD-collocated & 2 REFC @ $\Delta x=25 \mathrm{~m}$ & Explicit $v_{0}=75 \mathrm{~m}^{2} \mathrm{~s}^{-1}$ & 15.53 \\
SNFEM-LOR & 4 (REF) @ $\Delta x=25 \mathrm{~m}$ & Reference damping & 15.20 \\
SNFEM-LOR & 4 (REF) @ $\Delta x=25 \mathrm{~m}$ & Up-wind order 2 & 15.77 \\
SNFEM-LOR & 4 (REF) @ $\Delta x=25 \mathrm{~m}$ & Up-wind order 4 & 15.68 \\
\hline
\end{tabular}

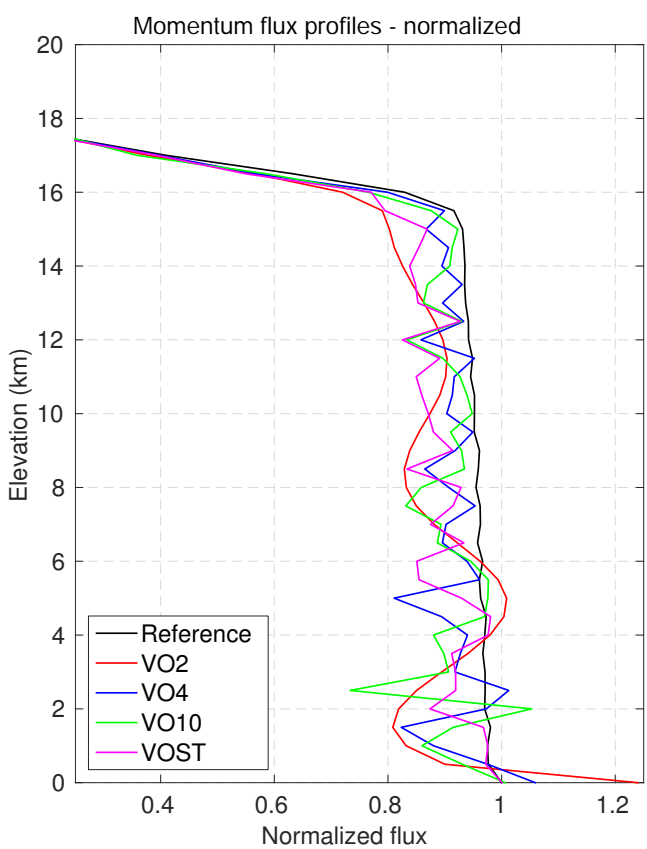

Figure 11. Schär mountain vertical profile of momentum flux for all experiments. The flux profiles are computed by $\int_{-X}^{X}\left\{\left[\bar{\rho}+\rho^{\prime}\right]\left[\bar{u}+u^{\prime}\right] w^{\prime}\right\} \mathrm{d} x$ at $t=10 \mathrm{~h}$ where overbars indicate initial condition values and primes are departures thereof. Results are interpolated to a regular $z$ coordinate with $\Delta z=500 \mathrm{~m}$ in experiments and reference solution to compute the integral flux. Results are normalized to the value at the surface in the reference solution.

second-order flow-dependent viscosity, but are closer to the reference solution with fourth-order diffusion. Both low- and high-order simulations show wave front positions that accurately approximate the reference results. However,

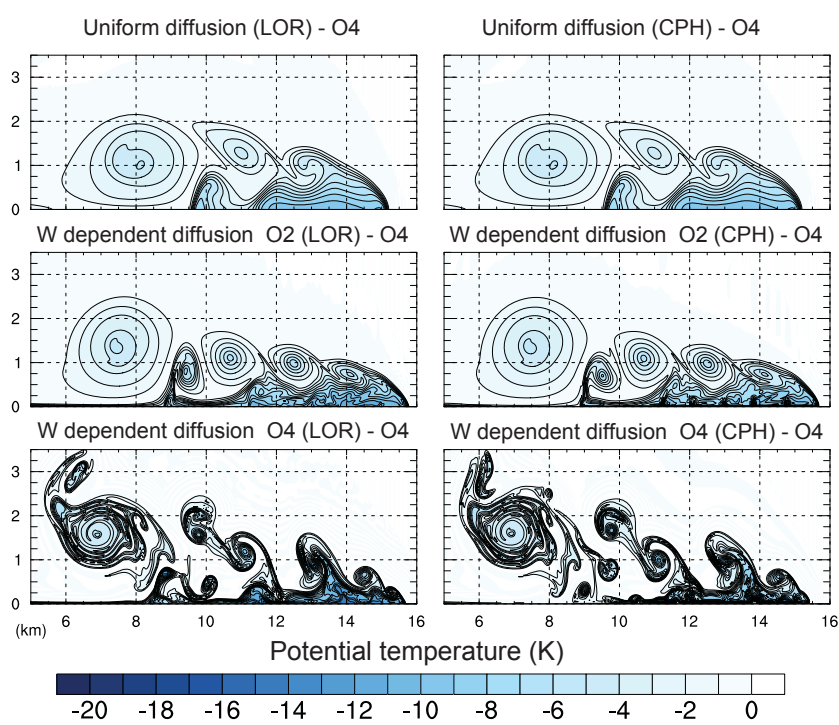

Figure 12. Straka density current test reference solutions at vertical order 4 in two staggering configurations LOR and CPH. Converged resolution of $\Delta x=25 \mathrm{~m}$ and $\Delta z=25 \mathrm{~m}$ shown. Vertical flow-dependent diffusion of order 2 and 4 (rows 2 and 3 ) is compared with the reference solution where an explicit second-order diffusion with $v_{0}=75 \mathrm{~m}^{2} \mathrm{~s}^{-1}$ is used (top row). Contour interval: $1.0 \mathrm{~K}$.

the structure of the Kelvin-Helmholtz rotors changes significantly with vertical order-of-accuracy and dissipation method shown in Fig. 13. The more scale-selective fourthorder flow-dependent viscosity shows greater detail in the structure of the rotors. In general, it is not recommended to use hyperdiffusion with a higher order than the dynamical 


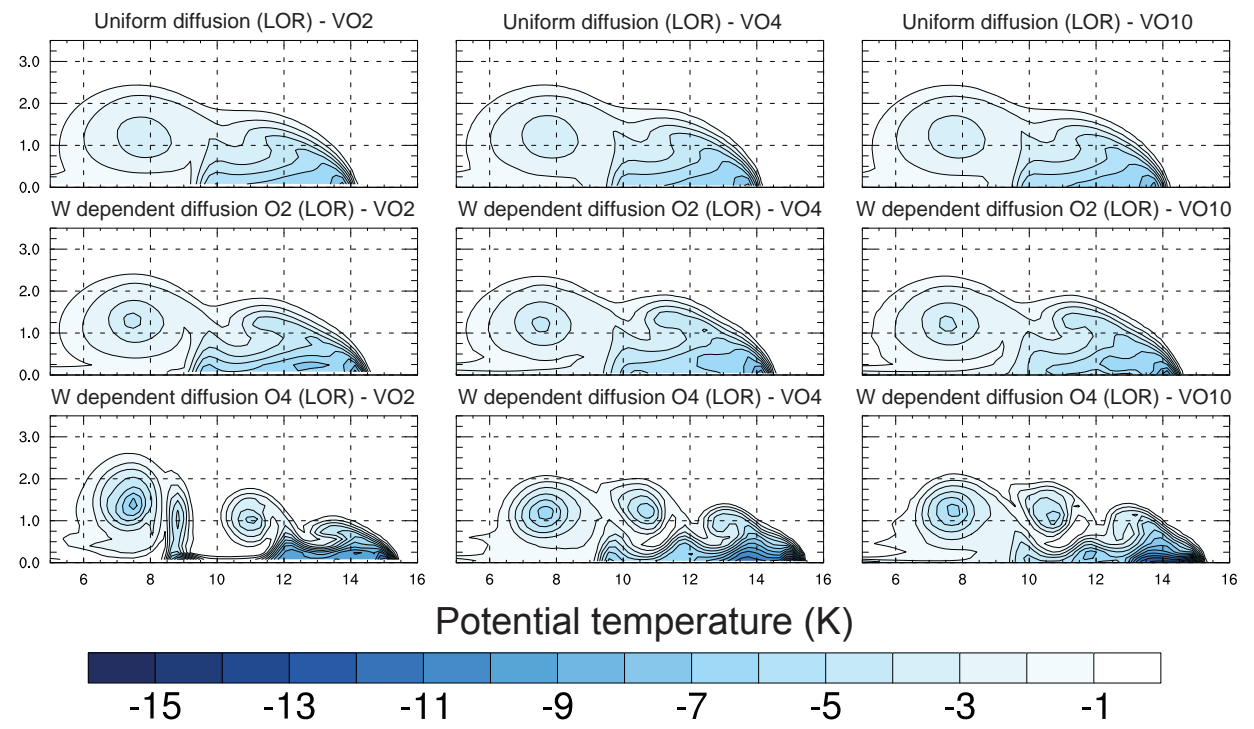

Figure 13. Straka density current test at vertical order 2, 4, and 10. Coarse, evaluation resolution of $\Delta x=190 \mathrm{~m}$ and $\Delta z=160 \mathrm{~m}$ shown. Vertical flow-dependent diffusion of order 2 and 4 (rows 2 and 3) is compared with the reference solution where an explicit second-order diffusion with $v_{0}=75 \mathrm{~m}^{2} \mathrm{~s}^{-1}$ is used (top row). Results for Lorenz (LOR) staggering shown. Contour interval: $1.0 \mathrm{~K}$.

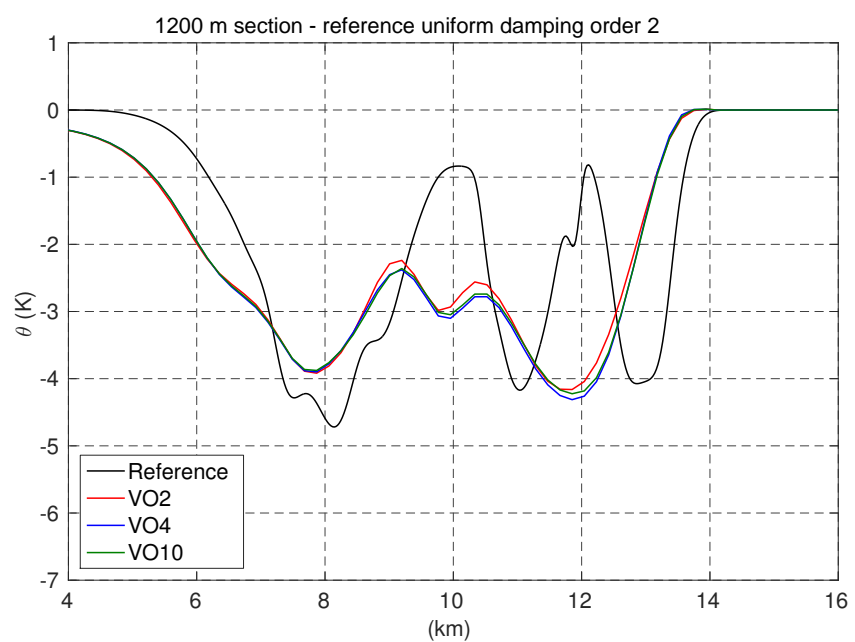

Figure 14. Straka density current test at vertical order 2, 4, and 10. Coarse, evaluation resolution of $\Delta x=190 \mathrm{~m}$ and $\Delta z=160 \mathrm{~m}$ with explicit second-order diffusion with $v_{0}=75 \mathrm{~m}^{2} \mathrm{~s}^{-1}$ compared at $1200 \mathrm{~m}$ with the reference solution $(\Delta x=25 \mathrm{~m}$ and $\Delta z=25 \mathrm{~m})$. Results for Lorenz (LOR) staggering shown.

discretization (bottom left) since more derivatives would be required than the polynomial space allows.

The use of flow-dependent hyperviscosity in second and fourth derivative order changes the structure of coarse experiments tending toward a three-rotor flow field shown in the reference solution as shown in Fig. 15. Curiously, the 10th-order vertical discretization with fourth-order flowdependent viscosity produces a flow that more closely approximates the reference solutions at a resolution that would otherwise be considered too poor for the dynamical features considered. However, the authors have not found a dynamical reason for correlation involving high-order vertical discretization coupled with high-order dissipation schemes and the reference solution with uniform damping.

Moreover, Fig. 15 indicates that magnitudes are significantly different for high-order dissipation cases. Wave front position at the $-1.0^{\circ} \mathrm{C}$ contour further given in Table 2 confirm that momentum fluxes are also captured more accurately as these are associated to the propagation speed of the wave front.

\subsection{Rising thermal bubble}

Thermal bubble experiments have become a standard in the development of non-hydrostatic mesoscale modeling systems. At very fine resolutions $(<10 \mathrm{~m})$, we test the ability to reproduce the simplest form of convection. This is a precursor to simulations of real atmospheric phenomena such as thunderstorms and other convective systems. A positive, symmetric perturbation to the potential temperature (buoyancy imbalance) causes a vertical acceleration that moves the bubble upward. Subsequently, shearing and compensating subsidence leads to two primary symmetrical eddies that further break down as the simulation progresses. We are interested in the evolution of the flow in terms of structure and conservative properties on $\theta$.

We present two flow scenarios: (a) the bubble rises and is allowed to interact with the top and lateral boundaries and (b) the so-called Robert smooth bubble experiment (as outlined in Giraldo and Restelli (2008)) that is a variation of the experiments of Robert (1993). In the former, the bubble 

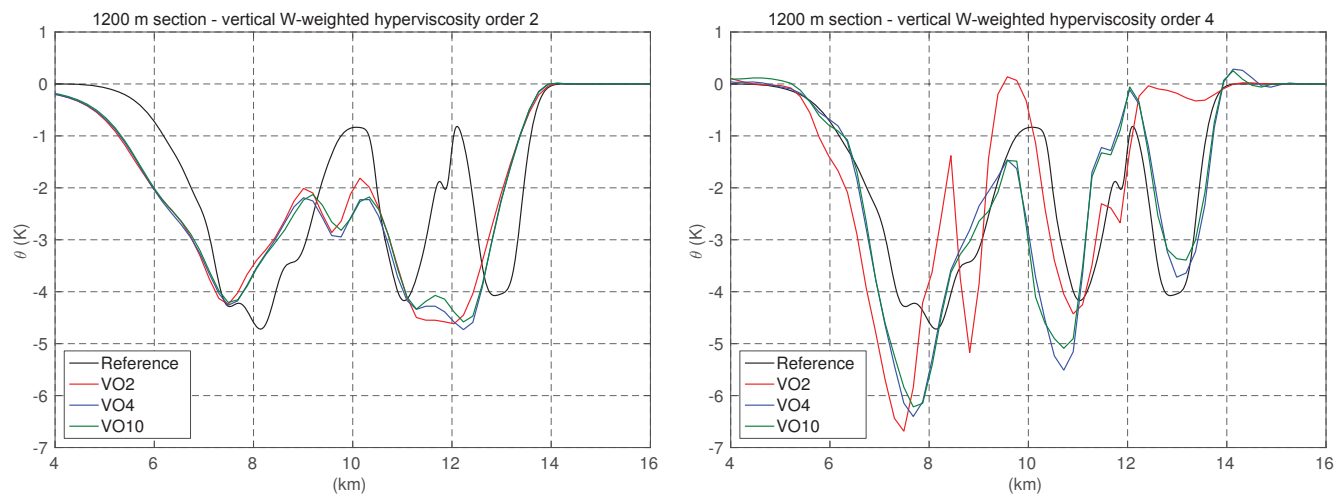

Figure 15. Straka density current test at vertical order 2, 4, and 10. Coarse, evaluation resolution of $\Delta x=190 \mathrm{~m}$ and $\Delta z=160 \mathrm{~m}$ shown. Vertical flow-dependent diffusion of derivative order 2 and 4 compared at $1200 \mathrm{~m}$ with the reference solution $(\Delta x=25 \mathrm{~m}$ and $\Delta z=25 \mathrm{~m})$. Results for Lorenz (LOR) staggering shown.

will meet the boundaries and develop shear instabilities and in the Robert bubble, shear instabilities develop in the interior of the flow. For these experiments, fourth-order viscosity is applied in the horizontal and vertical to the potential temperature and horizontal velocity fields. Furthermore, at finer resolutions we observe more fine-scale features of the thermal bubble, including tighter winding of the trailing edges at later times and sharper spatial gradients. Nonetheless, our comparisons for this test case are purely qualitative but remain consistent with previous results.

The background consists of a constant potential temperature field $\bar{\theta}=300 \mathrm{~K}$, with a small perturbation of the form

$\theta^{\prime}= \begin{cases}0 & \text { for } r>r_{c} \\ \frac{\theta_{c}}{2}\left[1+\cos \left(\frac{\pi r}{r_{c}}\right)\right] & \text { for } r \leq r_{c}\end{cases}$

where

$r=\sqrt{\left(x-x_{c}\right)^{2}+\left(z-z_{c}\right)^{2}}$.

Here we choose the amplitude and radius of the perturbation to be $\theta_{c}=0.5 \mathrm{~K}$ and $r_{c}=250 \mathrm{~m}$, respectively. The domain consists of a rectangular region $(x, z) \in[0,1000] \times$ $[0,1000] \mathrm{m}$ for the thermal bubble and $(x, z) \in[0,1000] \times$ $[0,1500] \mathrm{m}$ for the Robert bubble with $t \in[0,1200] \mathrm{s}$. The center-point of the bubble is located at $x_{c}=500 \mathrm{~m}$ and $z_{c}=$ $350 \mathrm{~m}$ for the thermal bubble and $z_{c}=260 \mathrm{~m}$ for the Robert bubble. The boundary conditions are no-flux over all boundaries. No Rayleigh layer is used, and Coriolis forces are set to zero.

The reference grid spacing is $\Delta x=5 \mathrm{~m}$ and $\Delta z=5 \mathrm{~m}$ with $\Delta t=0.005 \mathrm{~s}$. This is considered the reference resolution following Giraldo and Restelli (2008). Experiments are conducted at a relatively coarser resolution of $\Delta x=10 \mathrm{~m}$ and $\Delta z=10 \mathrm{~m}$ with $\Delta t=0.01 \mathrm{~s}$. The fourth-order scalar and vector (vorticity and divergence separately) diffusion coeffi-
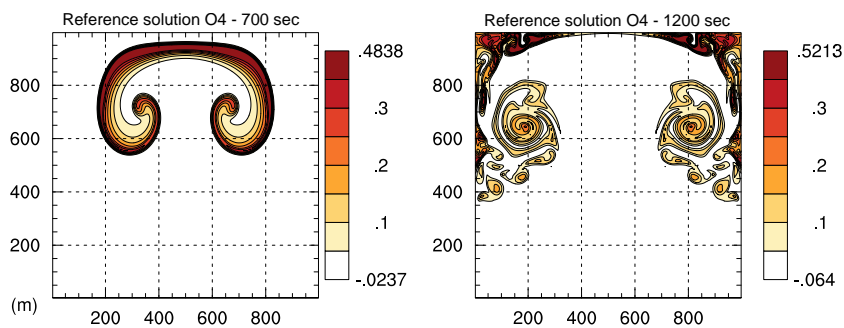

Figure 16. Rising thermal bubble potential temperature reference solution at vertical order 4 . Reference resolution $\Delta x=5 \mathrm{~m}$ and $\Delta z=5 \mathrm{~m}$. Flow at 700 and $1200 \mathrm{~s}$. Contour interval: $0.05 \mathrm{~K}$.

cients in are given by

$$
\begin{aligned}
v_{\text {scalar }} & =1.0 \times 10^{6} \mathrm{~m}^{4} \mathrm{~s}^{-1}, \\
v_{\text {vorticity }} & =1.0 \times 10^{6} \mathrm{~m}^{4} \mathrm{~s}^{-1}, \\
v_{\text {divergence }} & =1.0 \times 10^{6} \mathrm{~m}^{4} \mathrm{~s}^{-1} .
\end{aligned}
$$

The fourth-order scalar and vector (vorticity and divergence separately) diffusion coefficients are given by

$$
\begin{gathered}
v_{\text {scalar }}=1.0 \times 10^{6}\left(\frac{\Delta x}{L_{\text {ref }}}\right)^{3.2} \mathrm{~m}^{4} \mathrm{~s}^{-1}, \\
v_{\text {vorticity }}=1.0 \times 10^{6}\left(\frac{\Delta x}{L_{\text {ref }}}\right)^{3.2} \mathrm{~m}^{4} \mathrm{~s}^{-1}, \\
v_{\text {divergence }}=1.0 \times 10^{6}\left(\frac{\Delta x}{L_{\text {ref }}}\right)^{3.2} \mathrm{~m}^{4} \mathrm{~s}^{-1} .
\end{gathered}
$$

where $\Delta x$ is the element length in the $x$ direction and $L_{\text {ref }}=$ $1000.0 \mathrm{~m}$ is the reference length used for this test case.

Rising bubble experiments show the nonlinear dynamics of dry 2-D convection. The classic thermal bubble test shown in Fig. 16 shows potential temperature being advected conservatively throughout the domain at the reference resolution. These results use a dissipation mechanism that combines fourth-order hyperdiffusion of $\theta$ for horizontal modes 

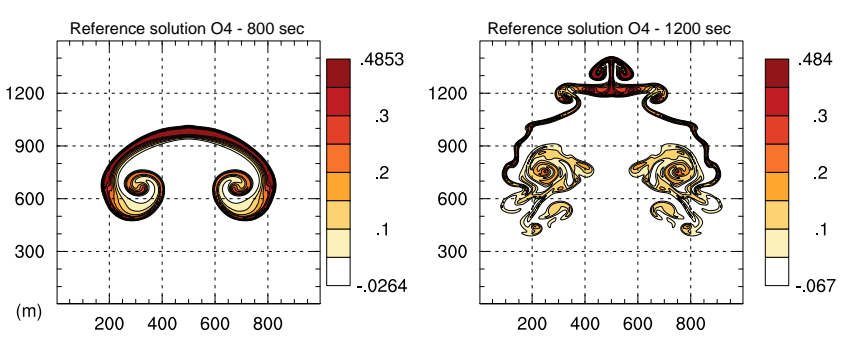

Figure 17. Rising Robert bubble potential temperature reference solution at vertical order 4 . Reference resolution $\Delta x=5 \mathrm{~m}$ and $\Delta z=5 \mathrm{~m}$. Flow at 800 and $1200 \mathrm{~s}$. Contour interval: $0.05 \mathrm{~K}$.

and scale-adaptive fourth-order flow-dependent hyperviscosity of $\theta$ for vertical modes. In this case, no diffusion is needed in the velocity or density fields to obtain a stable simulation.

The rising thermal bubble experiment is typically carried out and compared at 700 s precisely before the convective bubble interacts with the top boundary of the domain. We present this result for comparison with previous results in Fig. 18. However, it is also important to evaluate the conservative properties of the method and we carry out the simulation to 1200 seconds. Since Eq. (9) is a strict statement of constant potential temperature following fluid parcels, the results of Fig. 18 compared to Fig. 16 demonstrate that our method is stable and approximates conservation of $\theta$ closely when a high-order vertical discretization is used.

The Robert smooth bubble experiment extends the vertical domain allowing for the onset of Kelvin-Helmholtz instabilities in the flow. The solution at the reference resolution is shown in Fig. 17. The exact time and manner in which the instabilities arise is strongly dependent on the vertical order and dissipation method used in the simulation. In the reference solution, the onset of unstable eddies begins at approximately $900 \mathrm{~s}$ with the flow transitioning into vigorous mixing in the region of the primary rotors.

High-order vertical discretizations are typically associated with strong oscillations (Gibbs ringing) that can induce perturbations that can amplify turbulence, particularly if stabilization (such as upwinding or diffusion) is weak. The net effect is that a high-order vertical discretization, given the same horizontal discretization, changes the local mixing characteristics of the flow. This effect is seen clearly in Fig. 19. The 10th-order simulation has a structure that more closely approximates the reference result in Fig. 17. In the context of studies that seek to represent convective processes, we would expect entrainment fluxes to be improved at a coarser resolution with the higher-order vertical discretizations.

\subsection{Numerical characteristics of the method}

We briefly characterize the combined discretization strategy (horizontal spectral element, vertical SNFEM, and Strang IMEX) described in Sect. 3.3. We use the rising thermal bubble test (Sect. 4.4) to show that, overall, our method con-
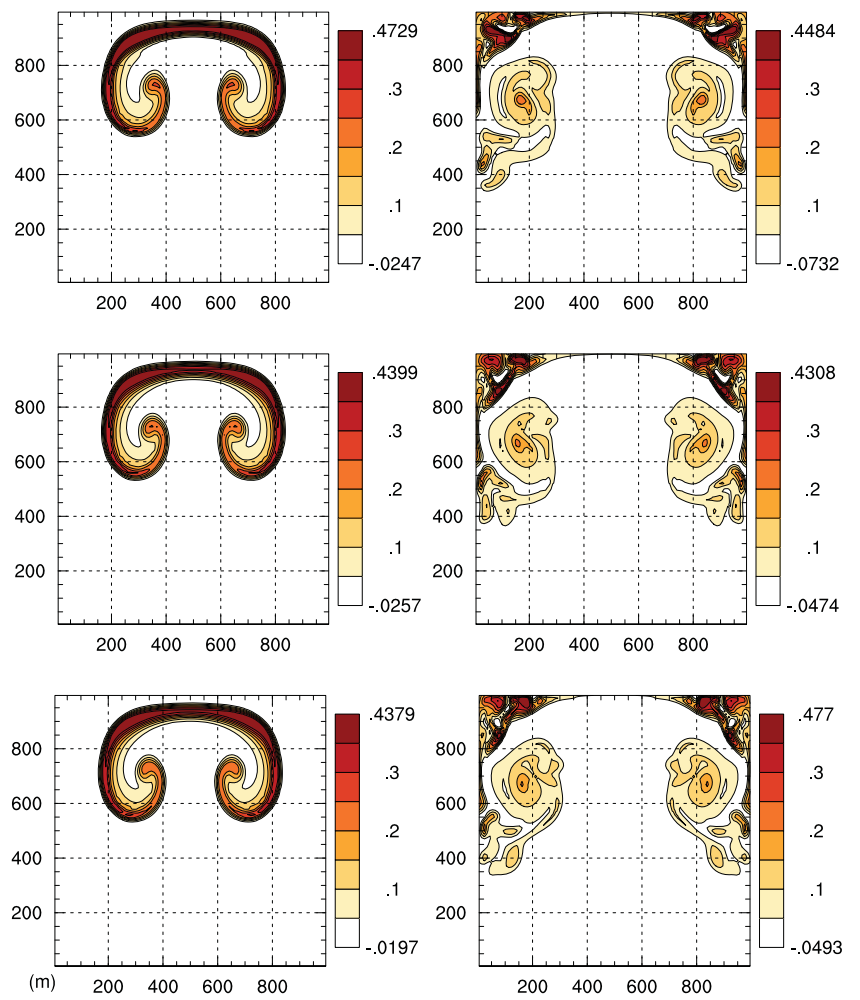

Figure 18. Rising thermal bubble potential temperature at vertical orders 2, 4, and 10. Convection bubbles at 700 and 1200 s. Coarse resolution $\Delta x=10 \mathrm{~m}$ and $\Delta z=10 \mathrm{~m}$. Extrema in $\theta$ shown. Contour interval: $0.05 \mathrm{~K}$

Table 3. Numerically computed estimates of the CourantFriedrichs-Lewy condition (maximum Courant number) using Thermal bubble tests over a wide range of horizontal : vertical aspect ratios. The maximum wave speed corresponds to the speed of sound given by $c=\sqrt{\gamma R_{d} T}$ where $\gamma=1.4, R_{d}=286.07$, and $T=300.5 \mathrm{~K}$.

\begin{tabular}{lrrr}
\hline Maximum Courant number & \multicolumn{3}{c}{ Aspect ratio $\Delta x: \Delta z$} \\
\cline { 2 - 4 } Vertical order & 1 & 10 & 100 \\
\hline 2 & 1.95 & 1.95 & 1.95 \\
4 & 1.95 & 1.95 & 1.86 \\
10 & 1.61 & 1.14 & 0.14 \\
\hline
\end{tabular}

verges at second order in space and time consistently across different vertical orders of accuracy as shown in Fig. 20. Theoretically, the maximal convergence rate for this test is at most second order in space since the $\theta$ perturbation is only continuous in its first derivative. Nonetheless, we observe sub-second-order convergence for the VO2 scheme applied to this test, driven by a loss of 1 order of accuracy from the use of vertical flow-dependent hyperviscosity (see Sect. 3.2.4). 

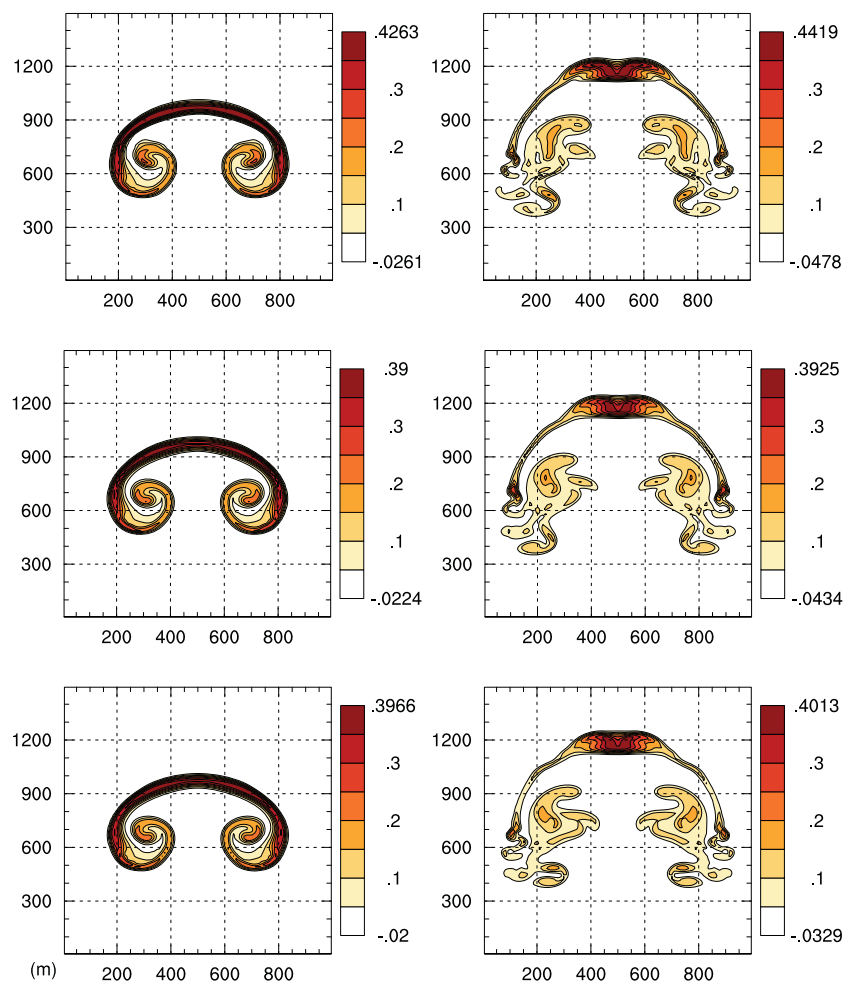

Figure 19. Rising Robert bubble potential temperature at vertical orders 2, 4, and 10. Convection bubbles at 800 at 1200 s. Coarse, evaluation resolution $\Delta x=10 \mathrm{~m}$ and $\Delta z=10 \mathrm{~m}$. Extrema in $\theta$ shown. Contour interval: $0.05 \mathrm{~K}$.

A numerically computed estimate of the CourantFriedrichs-Lewy (CFL) condition (maximum Courant number) as a function of grid spacing and element aspect ratio is given in Table 3 using the time integration technique outlined in Sect. 3.3. These results indicate a maximum Courant number of 1.95 at low order that degrades at higher aspect ratios and with higher vertical order. Moreover, all 2-D tests show a maximum Courant number of 1.95 while the 3-D Baroclinic wave test has a Courant number of 1.45. The theoretical CFL conditions for the spectral element discretization with temporal discretization (Eq. 56) are 2.12 and 1.49 for 1-D and 2$\mathrm{D}$ scalar advection, respectively. These results indicate that the operator split method as shown in Eqs. (49)-(52) combined with Strang integration allows 90 to $95 \%$ of the maximum time step possible using a consistent fourth-order space discretization. However, a more comprehensive evaluation of the theory underlying this CFL condition will be pursued in a future work due to changes observed with aspect ratio and vertical order of accuracy.

Furthermore, we show preliminary parallel performance scaling in Table 4 on a limited multi-core system. These results indicate a cost associated with denser element operations as vertical order of accuracy increases. However, more
Table 4. Thermal bubble test $(\Delta x=20 \mathrm{~m})$ average processor time taken per time step in seconds. Intel Core i7 4000 series under Linux with four computational cores on die (no interconnect hardware present). Results show relative scalability for Tempest using Message Passing Interface (MPI) architecture and IMEX partitioning with variable vertical order of accuracy. The implicit equations are solved using the GMRES with no preconditioner.

\begin{tabular}{lrrr}
\hline Computation time (s) & \multicolumn{3}{c}{ No. cores } \\
\cline { 2 - 4 } Vertical order & 1 & 2 & 4 \\
\hline 2nd & 0.117 & 0.070 & 0.061 \\
4th & 0.163 & 0.102 & 0.082 \\
10th & 0.248 & 0.143 & 0.106 \\
\hline
\end{tabular}

controlled experiments using a distributed platform will be conducted as our parallel implementation is optimized.

Plots of the normalized change in mass and energy, along with integrated zonal and vertical momentum from the Robert smooth bubble test (Sect. 4.4) are given in Fig. 21. As expected, total mass is conserved to near-machine precision. Total energy is not explicitly conserved by this method, so we observe small oscillations of total energy about its initial value. Note that although total energy is not non-increasing, it does not show exponential growth that would be characteristic of a linear instability, and remains bounded over the duration of the simulation. To ensure this result held for longterm simulations, the rising thermal bubble experiments were carried out to $1 \mathrm{~h}$, and revealed no sign of instability.

Further investigation of this issue seems to suggest roots in the way the stabilization mechanism interacts with the lateral boundaries, since the purely advective scheme with no stabilization shows nearly flat total energy. Consequently, we hypothesize this result may be associated with the inverse energy cascade from 2-D turbulence theory drawing energy from the unresolved scales in a limited manner. Note that the stabilization mechanisms described by this work (horizontal and vertical hyperviscosity), which work directly on the $\boldsymbol{u}$ and $\theta$ fields, do not act to explicitly diffuse energy; the strategy is intended to emphasize flow features. A more aggressive diffusion strategy could be implemented to ensure that energy does not increase at the cost of increased diffusive errors.

For a horizontally symmetric test such as the rising thermal bubble (anti-symmetric in $u$ ), one would expect that total zonal momentum is equal to zero over the duration of the simulation. However, we clearly observe deviations from symmetry by the end of the simulation. These violations of symmetry are associated with how the spectral element method is updated in the horizontal: since horizontal derivatives are computed in an inherently asymmetric manner, namely in the direction of increasing $x$, small differences on the order of machine epsilon appear between the solution $x<500 \mathrm{~m}$ and $x>500 \mathrm{~m}$. The oscillatory signal in the ver- 

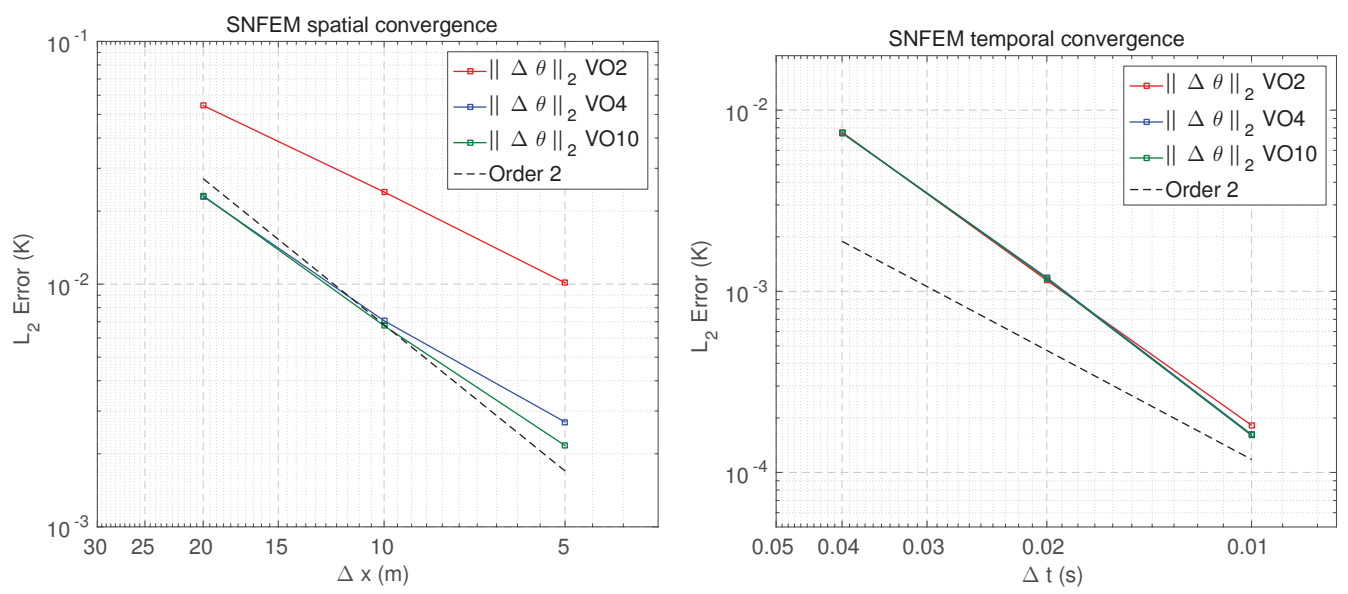

Figure 20. Spatial (left) and temporal (right) self convergence at various vertical orders of accuracy. Thermal bubble test at $200 \mathrm{~s}$. Spatial resolution for temporal convergence is $10 \mathrm{~m}$ with reference $\Delta t=0.001 \mathrm{~s}$. Reference spatial resolution is $\Delta x=2 \mathrm{~m}$.

tical momentum is attributed to strong vertically propagating sound waves that emerge from the initial perturbation being reflected by the no-flux boundary condition at the top and bottom of the model grid. Note that it is not expected that vertical momentum is conserved due to the presence of gravitational forcing.

\section{Conclusions}

The idea of separating the vertical and horizontal dynamics in atmospheric modeling systems has roots in the scale differences that characterize atmospheric flows. This principle has been fully exploited in the development of global and mesoscale models, along with the application of the hydrostatic approximation. This paper adds to the modern literature on modeling atmospheric dynamics by analyzing a novel discretization technique for achieving high-order accuracy in the vertical while maintaining the desirable properties of staggered methods. We refer to this technique as the staggered nodal finite-element method (SNFEM).

The test suite we present in this work is not exhaustive, but it is intended to evaluate the performance of the numerical schemes under conditions of near-hydrostatic synoptic-scale flow in Sect. 4.1, linear, mesoscale, non-hydrostatic flow with topography in Sect. 4.2, and fully nonlinear, non-hydrostatic, large-eddy simulation (LES) scale flow in Sect. 4.3 and Sect. 4.4. As global models progress into the regime of nonhydrostatic flows, real flow cases will be characterized by one or more of the properties mentioned, and likely in combination when variable or adaptive meshing methods are used. More importantly, we expect that uniform or mixed grids being prepared in research will begin to span the scale range that includes the transition to non-hydrostatic dynamics and on to large-eddy flows.
In general, we postulate that a higher-order method based on finite elements will be more accurate at a given resolution with minimal computational cost relative to a loworder method. Our results demonstrate that a high-order vertical coordinate approximates well-resolved reference results at coarser resolutions that would be otherwise considered poorly represented. Our experiments nonetheless are constrained by the order of horizontal and temporal discretizations. Therefore, we restrict our recommendation to the use of fourth-order SNFEM as optimum for the tests given here. In general the combined spatial order of accuracy should be consistent to maximize the effect of increased accuracy. The high-order approximation provides an improvement to the vertical dynamics and so reduces the need for higher vertical resolution. This benefit would prove effective when variablegrid methods are considered and nesting mesh levels can be saved by employing the SNFEM at high-order. The use of staggering in conjunction with high order has further benefits, in particular the avoidance of stationary computational modes that are known to persist with collocated methods.

However, there are some trade offs when increasing the vertical order: (1) for a vertically implicit method, fewer high-order elements lead to a dense matrix structure that is more expensive to invert, (2) the oscillatory nature of the polynomial functions that make up the interpolants within an element have physical consequences (involving nonlinear processes) at the smallest scales, and (3) higher-order spatial discretizations often require smaller time steps or higher order temporal discretizations. Figure 4 shows the times required for computations of varying vertical order and processor scaling. The results confirm that the relative cost in moving to fourth order is indeed modest relative to the use of higher orders.

The first point can be addressed in the construction of the software where parallelization and correct use of hardware resources minimizes the dense operations that high-order el- 

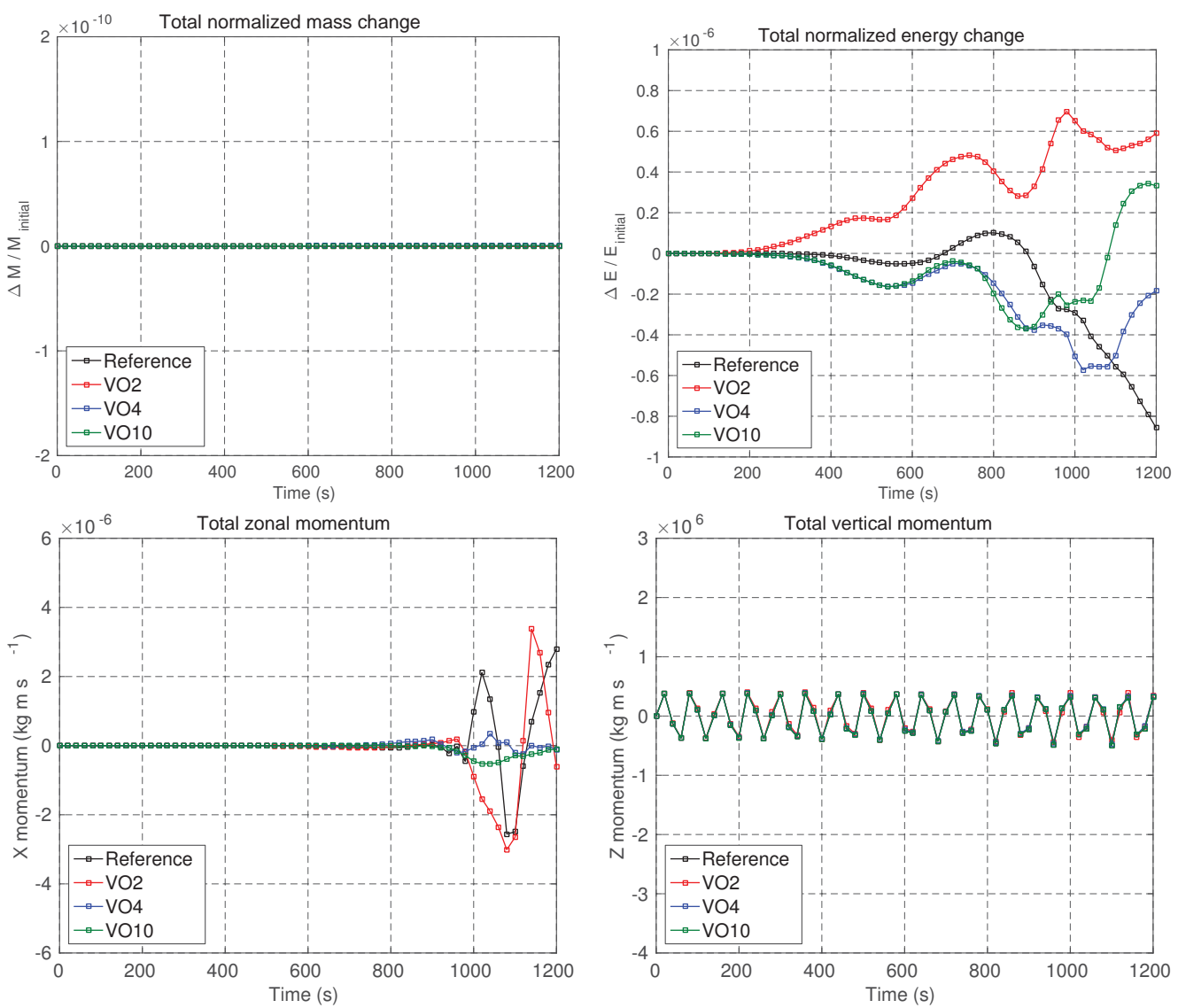

Figure 21. Observed normalized change in mass and energy (top row), along with zonal and vertical momentum (bottom row) using the Robert bubble experiment in Sect. 4.4. The reference solution corresponds to Fig. 17 with $5 \mathrm{~m}$ resolution. Evaluation experiments use variable vertical order (VO) SNFEM at $10 \mathrm{~m}$ resolution corresponding to Fig. 19. Total normalized mass and energy change are computed as $\left(Q_{\mathrm{t}}-Q_{\text {initial }}\right) / Q_{\text {initial }}$.

ements imply. We saw in Fig. 19 that oscillations associated with high-order interpolants helped to approximate fine-scale structures, but these oscillations can also be harmful depending on the flow condition. While vertical order of accuracy can be increased up to the total number of vertical levels, e.g., results from the Schär cases in Fig. 9, increasing computational expense indicates that intermediate orders of accuracy will generally be most effective. In this study, many of the results at fourth order sufficiently improve solutions relative to low-order alternatives.

Furthermore, when physical instabilities arise, a consistent, high-order, and scale selective dissipation strategy is necessary. In this regard, finite-element methods allow for the construction of diffusion operators for this purpose e.g., Sect. 3.2.3. We can experiment with different combinations of diffusion operators including coefficients that are variable in space. While scale-selective fourth-order operators with some grid resolution dependence are sufficient for this work, we intend to explore a wider range of strategies based on polynomial filtering, variational multi-scale methods, etc., with the goal of eliminating the tuning procedure associated with user-provided coefficients.

The numerical dissipation strategy implemented here serves two primary goals: (1) stabilization of the computations and (2) as a form of closure for the Euler equations solved on a truncated grid. The methods we employ allow for the construction of derivative operators of various orders in a consistent manner. Tempest features a system that allows for diffusion to be applied in a selective manner on variables that are split according to the time integration scheme.

Further experiments are necessary to test the extent of the third point above. For this work, we used a second-order Strang time integration scheme (Sect. 3.3) that was sufficiently robust to carry out all of the experiments up to 10th order without overly restricting time step size relative to the second-order simulations.

The authors conclude the following based on the experiments conducted and properties of the SNFEM:

1. Staggering has been generalized to finite-element methods combining continuous and discontinuous for- 
malisms. The result is a method that closely parallels the behavior of staggered finite differences eliminating stationary modes. This is strictly true for the lowest order finite elements and we restrict ourselves to observe that consistent behavior extends to high-order staggered elements pending a formal wave analysis.

2. Variable order of accuracy is an effective strategy that can compensate for limitations in grid-scale resolution. However, the effects at very high order must be understood and controlled with appropriate stabilization methods. In general, "intermediate" orders (about 4th order) are recommended with consideration for consistency in overall spatial order given an IMEX partitioned architecture

We emphasize that, while the equations are formulated in a coordinate-free manner, the results given all correspond to regular Cartesian coordinates as defined by the metrics in Eqs. (22) and (21). Experiments corresponding to small planet and global domains are left for a subsequent work. However, any curved geometry with a terrain-following surface topography can be applied to the equations since all grid information is held in the metric terms described in Sect. 2. As such, the effects of curved geometry and variable vertical order-of-accuracy are only addressed here in the Schär and Baroclinic wave cases (using the $\beta$ plane approximation). From a design perspective, metric terms are precomputed and derivative operators are built in the natural, local coordinate frame when any grid is used.

Tempest is constructed to provide a unified multi-scale platform for atmospheric simulation. Experiments can be carried out readily at all scales of importance from longterm climate simulations to high-resolution weather prediction. Development is underway to include moisture transport and phase transformations as well as to further improve time integration performance. Coupled with highly accurate, efficient, and robust methods to compute dynamics, Tempest will evolve to produce reliable precipitation forecasts as well as long-term climate simulations as part of the greater effort to understand the impending challenges brought on by rapid climate change.

\section{Code and data availability}

The Tempest codebase used to generate the results in this publication are available through the following Git repository: https://github.com/paullric/tempestmodel.

Acknowledgements. The authors would like to thank Hans Johansen, Mark Taylor, and David Hall for their assistance in refining this manuscript. Funding for this project has been provided by the Department of Energy, Office of Science project "A Nonhydrostatic Variable Resolution Atmospheric Model in ACME."

Edited by: S. Marras

\section{References}

Arakawa, A. and Moorthi, S.: Baroclinic instability in vertically discrete systems, J. Atmos. Sci., 45, 1688-1708, doi:10.1175/15200469(1988)045<1688:BIIVDS>2.0.CO;2, 1988.

Ascher, U., Ruuth, S., and Spiteri, R.: Implicit-explicit RungeKutta methods for time-dependent partial differential equations, Appl. Numer. Math., 25, 151-167, doi:10.1016/S01689274(97)00056-1, 1997.

Charney, J. and Phillips, N.: Numerical integration of the quasi-geostrophic equations for barotropic and simple baroclinic flows, J. Atmos. Sci., 10, 71-99, doi:10.1175/15200469(1953)010<0071:NIOTQG>2.0.CO;2, 1953.

Cotter, C. and Thuburn, J.: A finite element exterior calculus framework for the rotating shallow-water equations, J. Comput. Phys., 231, 7076-7091, 2012.

Cotter, C. and Thuburn, J.: Mixed finite elements for numerical weather prediction, J. Comput. Phys., 257, 1506-1526, 2014.

Dennis, J., Edwards, J., Evans, K. J., Guba, O. N., Lauritzen, P., Mirin, A. A., St-Cyr, A., Taylor, M. A., and Worley, P. H.: CAMSE: A scalable spectral element dynamical core for the Community Atmosphere Model, Int. J. High Perform. Comput. Appl., 26, 74-89, doi:10.1177/1094342011428142, 2011.

Gal-Chen, T. and Somerville, R. C. J.: On the use of a coordinate transformation for the solution of the Navier-Stokes equations., J. Comput. Phys., 17, 209-228, doi:10.1016/0021-9991(75)900376, 1975.

Giraldo, F. X. and Restelli, M.: A study of spectral element and discontinuous Galerkin methods for the Navier Stokes equations in nonhydrostatic mesoscale atmospheric modeling: Equation sets and test cases, J. Comput. Phys., 227, 3849-3877, doi:10.1016/j.jcp.2007.12.009, 2008.

Giraldo, F. X. and Rosmond, T. E.: A scalable Spectral Element Eulerian Atmospheric Model (SEE-AM) for NWP: Dynamical core tests, Mon. Weather Rev., 132, 133-153, doi:10.1175/15200493(2004)132<0133:ASSEEA>2.0.CO;2, 2004.

Giraldo, F. X., Kelly, J. F., and Constantinescu, E. M.: Implicitexplicit formulations of a three-dimensional nonhydrostatic unified model of the atmosphere (NUMA), SIAM J. Sci. Comput., 35, B1162-B1194, doi:10.1137/120876034, 2013.

Huynh, H.: A flux reconstruction approach to high-order schemes including discontinuous Galerkin methods, AIAA paper, 4079, 2007.

Kinnmark, I. P. and Gray, W. G.: One step integration methods with maximum stability regions, Math. Comput. Simulat., 26, 87-92, doi:10.1016/0378-4754(84)90039-9, 1984a.

Kinnmark, I. P. and Gray, W. G.: One step integration methods of third-fourth order accuracy with large hyperbolic stability limits, Math. Comput. Simulat., 26, 181-188, doi:10.1016/03784754(84)90056-9, 1984b.

Klemp, J. B., Skamarock, W. C., and Fuhrer, O.: Numerical Consistency of Metric Terms in Terrain-Following Coordinates, Mon. Weather Rev., 131, 1229-1239, doi:10.1175/15200493(2003)131<1229:NCOMTI>2.0.CO;2, 2003.

Lin, S.-J.: A "vertically Lagrangian" finite-volume dynamical core for global models, Mon. Weather Rev., 132, doi:10.1175/15200493(2004)132<2293:AVLFDC>2.0.CO;2, 2004.

Melvin, T., Staniforth, A., and Thuburn, J.: Dispersion analysis of the spectral element method, Q. J. Roy. Meteorol. Soc., 138, 1934-1947, doi:10.1002/qj.1906, 2012. 
Robert, C.: Bubble convection experiments with a semi-implicit formulation of the Euler equations, J. Atmos. Sci., 50, 1865-1873, doi:10.1175/1520-0493(2002)130<2459:ANTFVC>2.0.CO;2, 1993.

Schär, C., Leuenberger, D., Fuhrer, O., Luthi, D., and Girard, C.: A New Terrain-Following Vertical Coordinate Formulation for Atmospheric Prediction Models, Mon. Weather Rev., 130, 2459-2480, doi:10.1175/15200493(2002)130<2459:ANTFVC>2.0.CO;2, 2002.

Smith, R. B.: The influence of mountains on the atmosphere, Adv. Geophys., 21, 87-230, 1979.

Straka, J. M., Wilhelmson, R. B., Wicker, L. J., Anderson, J. R., and Droegemeier, K. K.: Numerical solutions of a non-linear density current: A benchmark solution and comparisons, Int. J. Numer. Meth. Fl., 17, 1-22, doi:10.1002/fld.1650170103, 1993.

Taylor, M. A. and Fournier, F. A.: A compatible and conservative spectral element method on unstructured grids, J. Comput. Phys., 229, 5879 - 5895, doi:10.1016/j.jcp.2010.04.008, 2010.

Thuburn, J.: Vertical discretizations giving optimal representation of normal modes: Sensitivity to the form of the pressuregradient term, Q. J. Roy. Meteor. Soc., 132, 2809-2825, doi:10.1256/qj.06.10, 2006.

Thuburn, J. and Woollings, T.: Vertical discretizations for compressible Euler equation atmospheric models giving optimal representation of normal modes, J. Comput. Phys., 203, 386-404, doi:10.1016/j.jcp.2004.08.018, 2005.

Toy, M. D. and Randall, D. A.: Comment on the article "Vertical discretizations for compressible Euler equation atmospheric models giving optimal representation of normal modes" by Thuburn and Woollings, J. Comput. Phys, 223, 82-88, doi:10.1016/j.jcp.2006.08.022, 2007.
Ullrich, P. and Guerra, J.: Exploring the Effects of a HighOrder Vertical Coordinate in a Non-Hydrostatic Global Model., Conf. on Computational Science: Numerical and Computational Developments to Advance Multiscale Earth System Models (MSESM), 2015.

Ullrich, P. A.: A global finite-element shallow-water model supporting continuous and discontinuous elements, Geosci. Model Dev., 7, 3017-3035, doi:10.5194/gmd-7-3017-2014, 2014a.

Ullrich, P. A.: Understanding the treatment of waves in atmospheric models. Part 1: The shortest resolved waves of the 1D linearized shallow-water equations, Q. J. Roy. Meteor. Soc., 140, 14261440, doi:10.1002/qj.2226, 2014 b.

Ullrich, P. A. and Jablonowski, C.: MCore: A non-hydrostatic atmospheric dynamical core utilizing high-order finitevolume methods, J. Comput. Phys., 231, 5078-5108, doi:10.1016/j.jcp.2012.04.024, 2012.

Ullrich, P. A., Reed, K. A., and Jablonowski, C.: Analytical initial conditions and an analysis of baroclinic instability waves in $f$ - and $\beta$-plane 3D channel models, Q. J. Roy. Meteor. Soc., 141,2972-2988, doi:10.1002/qj.2583, 2015.

Zienkiewicz, O., Taylor, R., and Zhu, J.: The finite element method: its basis and fundamentals, Vol. 1, Butterworth-Heinemann, 2005. 